\title{
A probabilistic approach for high cycle fatigue of Wire and Arc Additive Manufactured parts taking into account process-induced pores
}

\author{
Lorenzo Bercelli $^{1}$, Sylvain Moyne ${ }^{1}$, Matthieu Dhondt ${ }^{1}$, Cédric Doudard $^{1}$, Sylvain Calloch $^{1}$, Julien Beaudet $^{2}$ \\ ${ }^{1}$ ENSTA Bretagne, UMR CNRS 6027, IRDL, 29200 Brest, France \\ ${ }^{2}$ Naval Group Research, Technocampus Océan, 5 rue de l’Halbrane, 44340 Bouguenais, France
}

\begin{abstract}
Wire and Arc Additive Manufacturing (WAAM) is a direct-energy deposition technique (unlike SLM or EBM) that builds up a part in a layer-by-layer fashion, each layer being constituted of interlaced weld beads. It is the best suited Additive Manufacturing (AM) technique for large structures thanks to its high deposition rate $(5 \mathrm{~kg} / \mathrm{h})$. The resulting material shows a rough surface, strong residual stress induced by its complex thermal history, a heterogeneous microstructure marked by the different weld passes as well as defects formed by gas pockets. Despite their rarity, pores are found to have a first-order influence on the fatigue life of machined specimens. The discrepancy in their size $(>100 \mu \mathrm{m})$ and position is responsible for a considerable scatter that makes classical fatigue tests ineffective. The aim of this study is to propose a novel approach to take into account the effect of rare WAAM-induced defects in high cycle fatigue. To achieve this, numerical porous structures are generated from the knowledge of the real pore population determined by tomography. Their fatigue performances are predicted via a two-scale probabilistic model identified on experimental self-heating results, on which pores have no influence. In that sense, the probabilistic model describes the behaviour of a virtually healthy material. Then, by computing a database of representative pore cases, the whole bundle of Wöhler curves for each numerical porous structure is determined. Finally, the numerical fatigue scatter is in close agreement with experimental data, and it is shown that the ranking in pore criticality according to the model matches the fractography observations.

Keywords: Wire and Arc Additive Manufacturing (WAAM); High cycle fatigue; Pores; Self-heating; Poisson point process
\end{abstract}

\section{Introduction}

Metal additive manufacturing (AM) allows for unprecedented design features such as hollow bodies, topologically optimized shapes [1] or even lattice structures [2], all of which can hardly be achieved through more classical processes such as casting or machining. It is also regarded as a production cost-saving technique since as-built AM parts are near net shape, reducing the amount of finish operations. Among the many available AM techniques, Wire and Arc Additive Manufacturing (WAAM) is the best suited to large structures thanks to its high material deposition rate $(5 \mathrm{~kg} / \mathrm{h} \mathrm{[3]})$. In contrast to powder-based processes, WAAM is a direct energy deposition technique: a wire input is melted by an arc onto a substrate, building up the part in a layer-by-layer fashion, each layer being constituted of interlaced weld beads. A typical production unit is composed of a 6-axis robot equipped with a welding torch, a power source and clamping tooling. Since the raw material is regular welding wire, WAAM is regarded as a cost-competitive production process [4].

The use of WAAM to produce structural parts in industry is not widespread yet. Extensive work is required to characterize the resulting material in terms of mechanical fatigue to give better confidence in its use. Indeed, AM materials are known to be highly sensitive to process parameters. The microstructure shows strong heterogeneities induced by the layer-by-layer building of the part $[5,6]$. As-built parts display high surface roughness, which is detrimental to fatigue performances [7-9]. Given the complex thermal history of AM parts, a strong residual stress field is induced [10,11]. Finally, some defects like lack of fusion or gas pores are formed depending on the process parameters and matter input $[12,13]$. For most metal AM technologies, pores are found to have a first-order influence on fatigue life, inducing large scatter of fatigue data of machined specimens [13-17].

The influence of powder-based AM defects on fatigue has been the subject of extensive research in the past three years. In Selective Laser Melting (SLM), it is shown through a crack propagation approach, that fatigue scatter is correlated with the geometry of pores, dismissing the influence of differences in microstructure [18]. The predictions of Murakami [19] or the Danninger-Weiss [20] models are explored in the case of SLM [21] as well as for Electron Beam Melting (EBM) [22], highlighting the fundamental influence on

Abbreviations: AM: Additive Manufacturing, EBM: Electron Beam Melting, FEA: Finite Element Analysis, RVE: Representative Volume Element, SEM: Scanning Electron Microscope, SLM: Selective Laser Melting, SNP: Stress - Number of cycles - Probability of failure, WAAM: Wire and Arc Additive Manufacturing

${ }^{*}$ Corresponding author. E-mail address: lorenzo.bercelli@ensta-bretagne.org 
fatigue life of the position of an isolated pore over its size. From this observation a novel approach is proposed in [16], where the total plastic zone in the vicinity of a defect is considered from Finite Element Analysis (FEA). Although gas pores found in WAAM parts have much larger dimensions $(>100 \mu \mathrm{m})$ and have smooth near-spherical shapes [23-25], to which notch fatigue and crack initiation approaches are best suited [26,27].

In the case of WAAM, a low density of defects can be achieved using the Cold Metal Transfer (CMT) technology [28,29]. Typical WAAM defects are round smooth pores, with sizes ranging from a few micrometers to more than half a millimeter [23, 30]. Fatigue samples extracted from optmized WAAM blocks show rare isolated pores with an important discrepancy in size and distance to free surface. As a result, a considerable scatter in fatigue data is observed [31] (for example compared to casting materials), posing quite a challenge to the assessment of fatigue properties by conventional methods (ASTM E466-15 standard [32], the staircase method [33]). These samples do not constitute a Representative Volume Element (RVE) of the material's fatigue behaviour: each sample can be seen as a unique structure. This is to be opposed to the case of casting for which the higher density of defects allows for repeatable fatigue results [34]. A single WAAM fatigue sample is not representative of the whole spectrum of possible pore cases. Moreover, samples are extracted from an initial deposited volume; this process randomizes the occurence of a pore case. In that regard, the approach proposed by S. Romano et al. [35] for SLM materials, where the occurence of killer defects is probabilized, is not suited for this WAAM material. Another methodology is developed in [36] where the choice is made to model fatigue scatter using a Weibull distribution identified on experimental data. Then, the fatigue life relative to each defect in a sample is calculated using a crack propagation approach, and ranked by likelihood according to the Weibull model. In the case of WAAM, the identification of a Weibull model on fatigue data would require extensive and cost-prohibitive testing. In light of this, it appears that a novel approach is required to predict the fatigue properties of a WAAM material with isolated pores.

The strategy of the present methodology is to generate a set of numerical structures with a realistic WAAM pore population and to forecast their individual fatigue performances. The objective is to compute a numerical scatter comparable to experimental observations. The different steps to the application of this methodology are illustrated in Figure 1:

(1) The behaviour in self-heating under cyclic loading of the WAAM material is determined through experimental tests [37-39], for which the diversity in rare pores has no influence.

(2) A probabilistic fatigue model is identified using the self-heating tests results [40,41]. As no influence of pores is considered, the fatigue behaviour of a defect free WAAM material is determined.

(3) Multiple material fragments are analyzed by tomography. A pore density and a diameter distribution law are identified.

(4) The impact of specific pore cases on fatigue is predicted by modeling porous structures in finite elements. Their constitutive material fatigue properties are determined by the probabilistic model. The calculation results of each pore case constitute a database for further fatigue forecasts.

(5) A draw of random numerical porous structures is performed: from the knowledge of the pore population, a numerical volume is populated with defects and cylinders are extracted from this volume.

6 The Stress - Number of cycles - Probability (SNP) curve of each numerical structure is interpolated from database values.

7 The bundle of SNP curves is validated by comparison with experimental fatigue results independent of the self-heating tests.

8 For each numerical structure, the critical pore can be determined. A population of critical defects is identified.

(9) The numerical critical population is validated by comparison with fractographies of the fatigue test campaign.

This paper is divided into three main sections. First, the experimental results of a bronze-aluminium WAAM material are presented. Microstructural observations, quasi-static mechanical performances and conventional fatigue results are exposed. The tomography analysis and its related distribution law are detailed, as well as the self-heating tests protocol and results. Secondly, the probabilistic model, predicting fatigue performances based on the self-heating behaviour, is thoroughly described. Thirdly, the application of the model in the case of a porous structure, the parametrization of a pore case and the generation of numerical samples are unfolded. Experimental results are used to validate the approach forecast.

\section{Material and experimental results}

The material is built from $C u A l 9 N i 5 F e 4(\mathrm{Wt} \%)$ bronze-aluminium welding wires. It was provided in the form of multi bead heat-treated blocks produced by Naval Group of the approximate dimensions $265 \mathrm{~mm}$ x $220 \mathrm{~mm}$ x $70 \mathrm{~mm}$ (Figure 2). The blocks were deposited using an intensity of $156 \mathrm{~A}$ and a voltage of $13.4 \mathrm{~V}$. The deposition and heat treatment parameters were identical for each block.

All of the mechanical samples were extracted vertically, along the building direction (or $z$ ). It is worth noting that all samples are indeed extracted from an initial volume, and not built as near-net shape samples like it is done with powder-based processes. Firstly, this means that no influence of the as-built rough surface is considered in the present study. Secondly, there is no particular reason for 


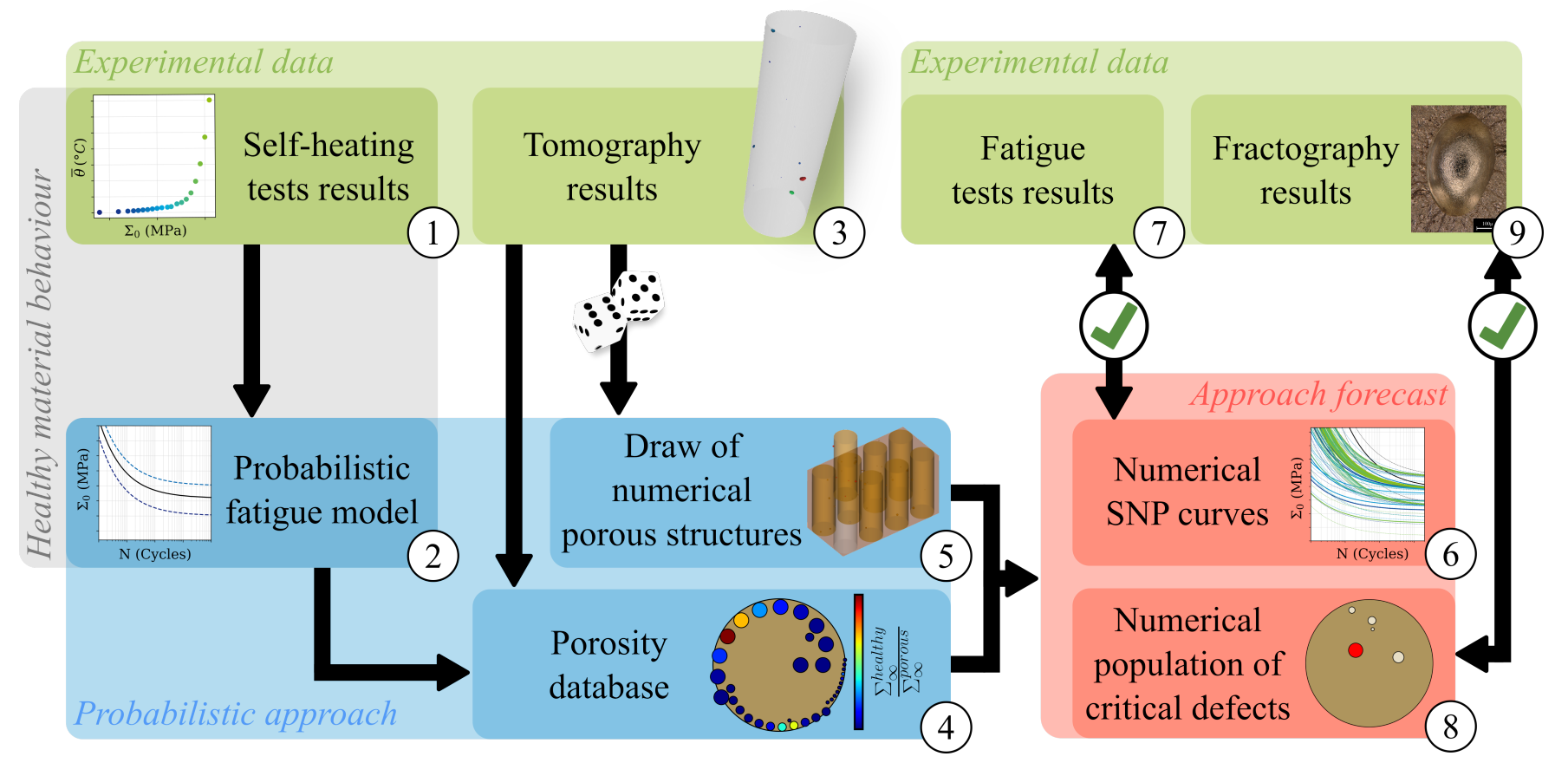

FIgURE 1 - Methodology diagram as detailed in the introduction

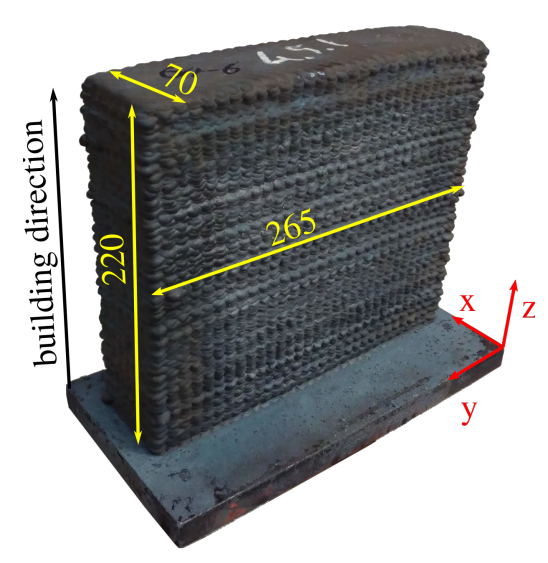

(a)

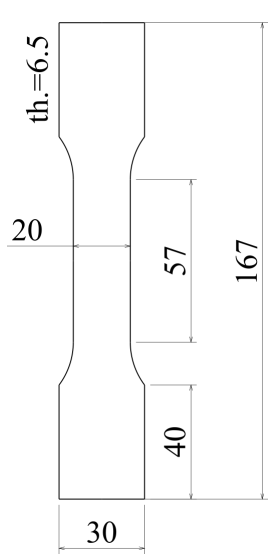

(b)

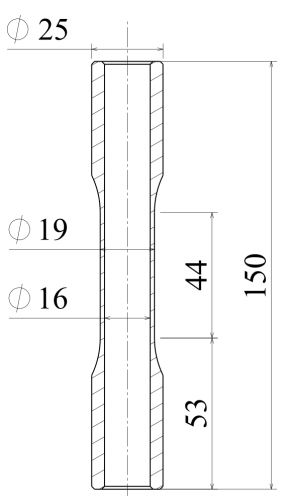

(c)

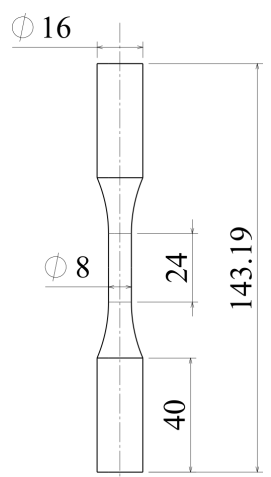

(d)

FIGURE 2 - Photo of a WAAM block (a), dimensions (in mm) of a quasi-static flat test sample (b), multiaxial self-heating test sample (c) and uniaxial self-heating sample (d)

a pore to be found in sub-surface, as samples are extracted from the core of the blocks.

\subsection{Microstructure}

The microstructure of additively manufactured metals has been thoroughly studied in literature. Both for powder-based and directenergy deposisiton technologies, the microstructure shows regular patterns of heterogeneities corresponding to the different building layers $[5,6,42,43]$. In the case of WAAM, the different weld beads can be found in the microstructure resulting in patterns of a few millimeters [44] as shown in Figure 3. The observed face is in the $(x ; z)$ plane (Figure 2). Observation samples were polished using a Struers LaboForce-100, following the procedure recommended by Struers for copper alloys, up to a $1 / 4 \mu \mathrm{m}$ diamond suspension. Specimens were etched for optical observations with the following reagent: $100 \mathrm{~mL}$ of water, $20 \mathrm{~mL}$ of hydrochloric acid and $5 \mathrm{~g}$ of iron(III) chloride. An additional polishing step using an active oxide polishing suspension was performed in lieu of etching for Scanning Electron Microscope (SEM) observations.

It appears that the boundary between two weld beads is marked by a discontinuity in grain size. Observing the microstructure with a SEM, we see it is dominated by an $\alpha$ phase with multiple precipitates (Figure 4). These precipitates are distinguished by their morphologies and chemical composition, as detailed in Table 1, taken from [45]. Fine $k_{4}$ precipitates appear inside the grains [46]. 


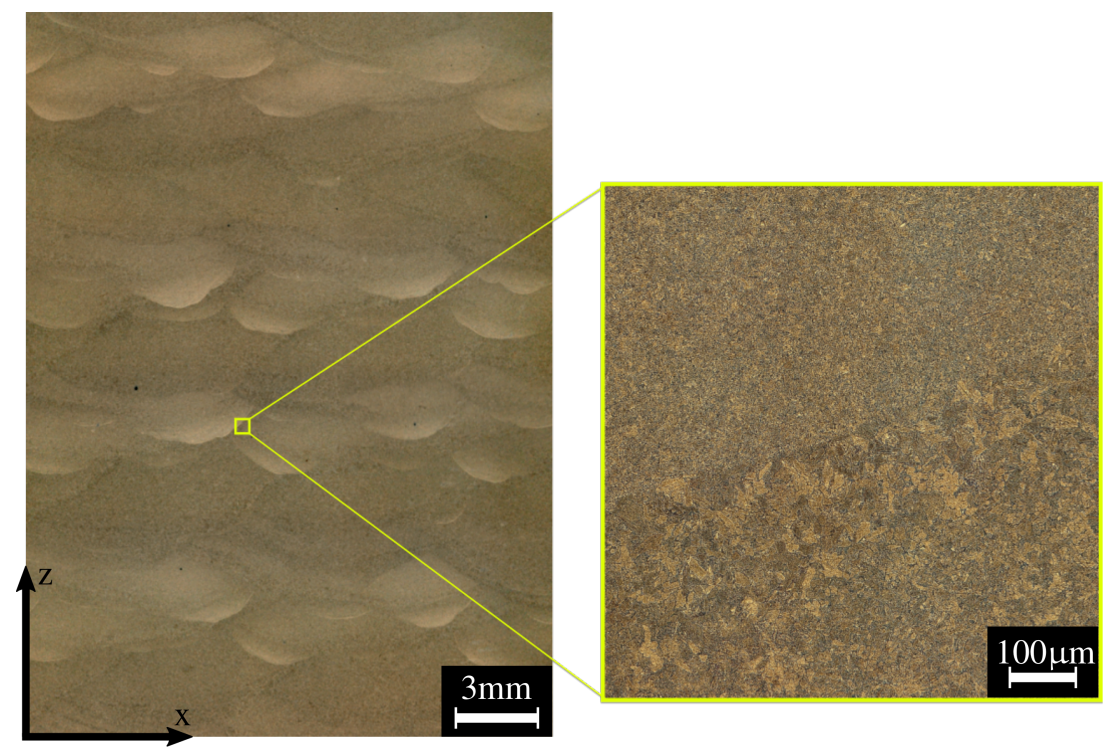

Figure 3 - Macrograph of the material microstructure and close up at the intersection between two weld beads

Lamellar Ni rich precipitates at the grain boundaries are identified as $k_{3}$, which can also be of globular form because of the heat treatment [45]. No $k_{2}$ precipitates are to be found, neither are observed large rosette like precipitates $k_{1}$ [47]. The fragile phase $\beta^{\prime}$ (or retained $\beta$ ) is not present, instead a $\alpha+k_{3}$ phase is formed during the heat treatment [48].

The grains in the WAAM material are globally much finer $(<50 \mu \mathrm{m})$ than in its as-built casting counterpart, in which some grains are longer than $100 \mu \mathrm{m}$ [49].

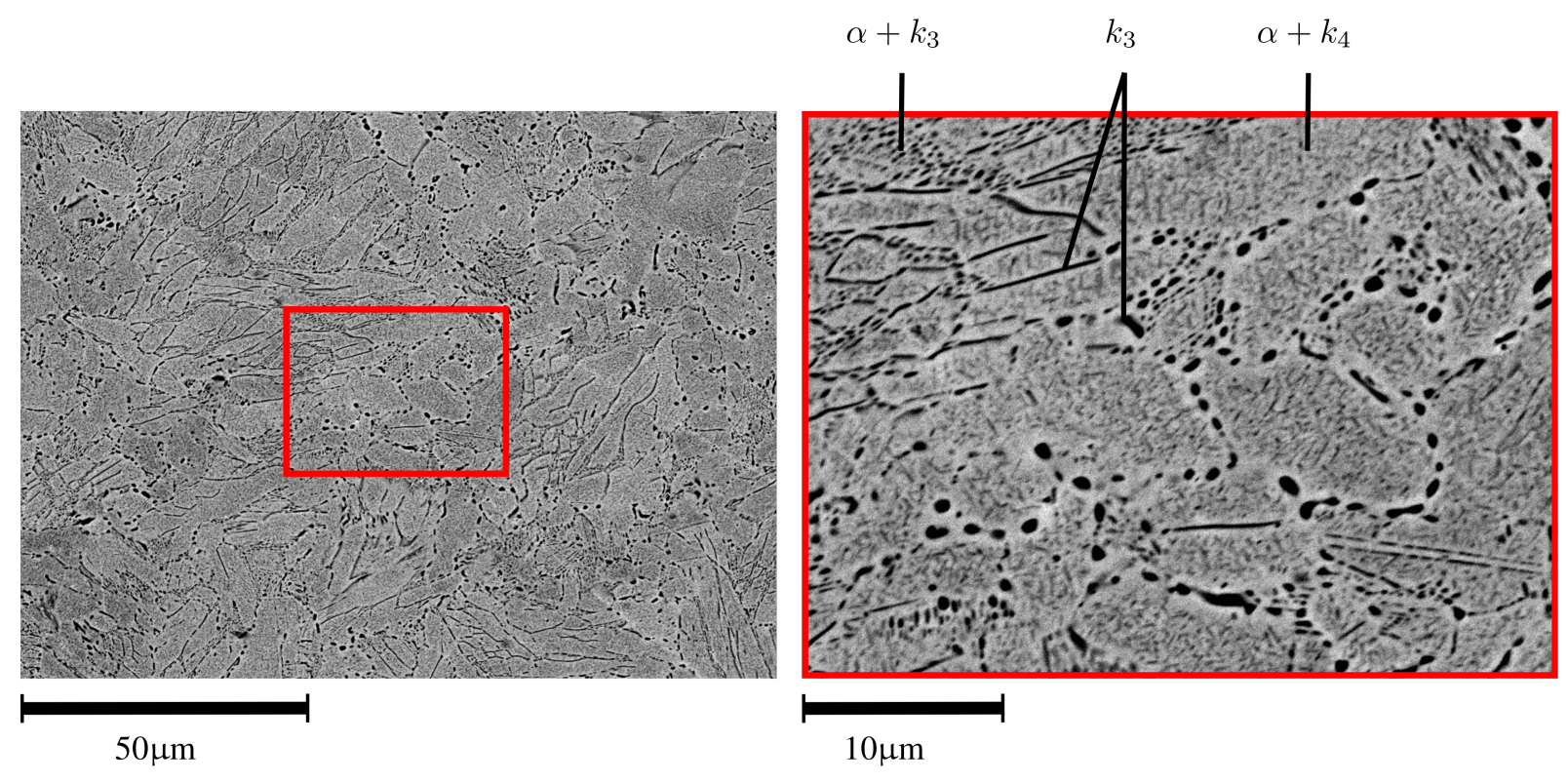

FIGURE 4 - SEM view of the microstructure

To make sure the microstructural heterogeneities did not impact the experimental results, care was taken in the dimensioning of the samples. A sufficient number of weld beads must be contained in a sample to make sure it constitutes an RVE in the sense of the microstructure.

Despite the knowledge that such microstructural heterogeneities have an influence on the stress field in the elastic domain [50], pores are found to be of primary importance in the fatigue life of AM materials [18]. For this reason, a more detailed study of the influence of microstructure on fatigue was not performed. 


\begin{tabular}{|c|c|c|c|c|c|}
\hline Phase & $\mathrm{Cu}(\mathrm{wt} \%)$ & $\mathrm{Al}(\mathrm{wt} \%)$ & $\mathrm{Ni}(\mathrm{wt} \%)$ & $\mathrm{Fe}(\mathrm{wt} \%)$ & $\mathrm{Mn}(\mathrm{wt} \%)$ \\
\hline$\alpha$ & 86.41 & 7.90 & 2.91 & 2.58 & 0.20 \\
\hline$\beta$ & 86.19 & 8.51 & 2.58 & 2.20 & 0.52 \\
\hline$k_{1}$ & 27.64 & 17.53 & 18.07 & 35.69 & 1.25 \\
\hline$k_{2}$ & 27.34 & 19.09 & 26.04 & 26.60 & 0.93 \\
\hline$k_{3}$ & 41.03 & 18.87 & 26.80 & 12.86 & 0.45 \\
\hline$k_{4}$ & 13.01 & 8.12 & 35.32 & 42.70 & 0.84 \\
\hline
\end{tabular}

TABLE 1 - Chemical composition of the bronze aluminium phases and precipitates from [45]

\subsection{Mechanical properties}

As a first step to characterize the WAAM material, quasi-static tension tests were performed. Flat samples (Figure $2 \mathrm{~b}$ ) were extracted from a block along its building direction. The transversal direction was not considered, as it was observed in different WAAM materials that there is little to no mechanical anisotropy at the macroscopic scale [5,51,52]. The tests were performed on an MTS tension-torsion servo-hydraulic test rig with a capacity of $250 \mathrm{kN}$. The tensile curves in normalized true stress and strain are shown in Figure 5. The WAAM material was compared to the corresponding as-built casting grade. The additively manufactured bronze-aluminium shows considerably greater values of elastic yield $\Sigma_{y}$, tensile strength $\Sigma_{U T S}$ and failure deformation $A_{\%}$ (Table 2). This is coherent with the observations of a finer microstructure with no brittle phase.

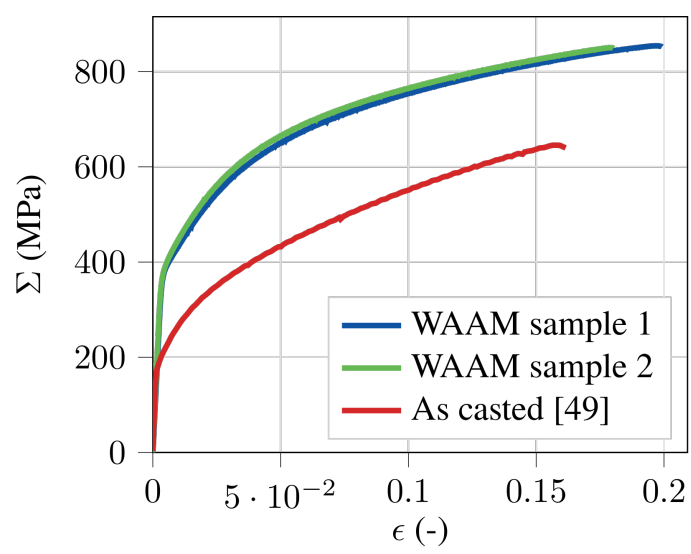

Figure 5 - Quasi-static tension curves in true stress and strain

Fractographies did not reveal defect-related failures in quasi-static tensile tests.

\begin{tabular}{|c|c|c|c|c|}
\hline Material & $\mathrm{E}(\mathrm{GPa})$ & $\Sigma_{y}(\mathrm{MPa})$ & $\Sigma_{U T S}(\mathrm{MPa})$ & $\mathrm{A}_{\%}(\%)$ \\
\hline WAAM & 113 & 394 & 852 & 19 \\
\hline Casting & 120 & 203 & 647 & 16 \\
\hline
\end{tabular}

TABLE 2 - WAAM and cast bronze aluminium static properties

\subsection{Fatigue}

Fatigue tests were performed on 46 cylindrical samples $8 \mathrm{~mm}$ in diameter and with a gauge length of $17.2 \mathrm{~mm}$ using a vibrophore. Stress amplitudes from 180MPa to 325MPa were explored at a stress ratio of $R=\frac{\Sigma_{\min }}{\Sigma_{\max }}=-1$ (zero mean stress). Tests were performed at a frequency of $100 \mathrm{~Hz}$ according to the standards NF A03-400 [53] and NF A03-401 [54]. A number of 2.5.107 cycles was chosen as a run-out criterion. The results are plotted in Figure 6.

It appears that the WAAM material has fatigue properties superior to its as-cast counterpart [49], although a very large scatter is observed in the WAAM fatigue results: for a same stress level, a ratio in fatigue life of more than 100 can be found. A fractography analysis of the samples sheds light on the fatigue scatter.

While a few samples show fatigue cracks initiating from the machined surface (green dots in Figure 7), $70 \%$ of the samples failed prematurely because of cracks initiating from a pore (blue dots in Figure 7). The presence of a pore in a sample acts as a stress 


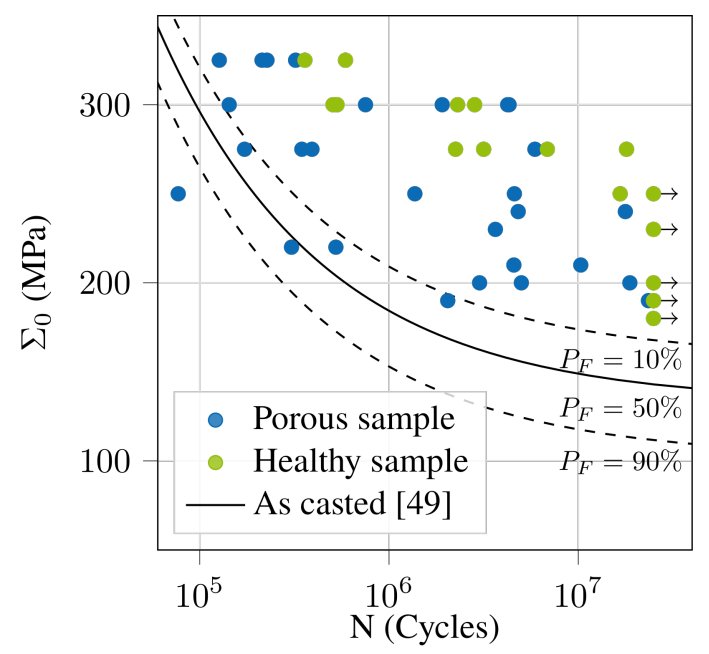

FIgURE 6 - Fatigue tests results at $R=-1$ of the WAAM material compared to its casted counterpart at various probabilities of failure

concentrator. The size and the distance to surface of the defect have an influence on the intensity of this concentration [55]. As a result, the scatter in size and position of the pores causes the scatter in fatigue.

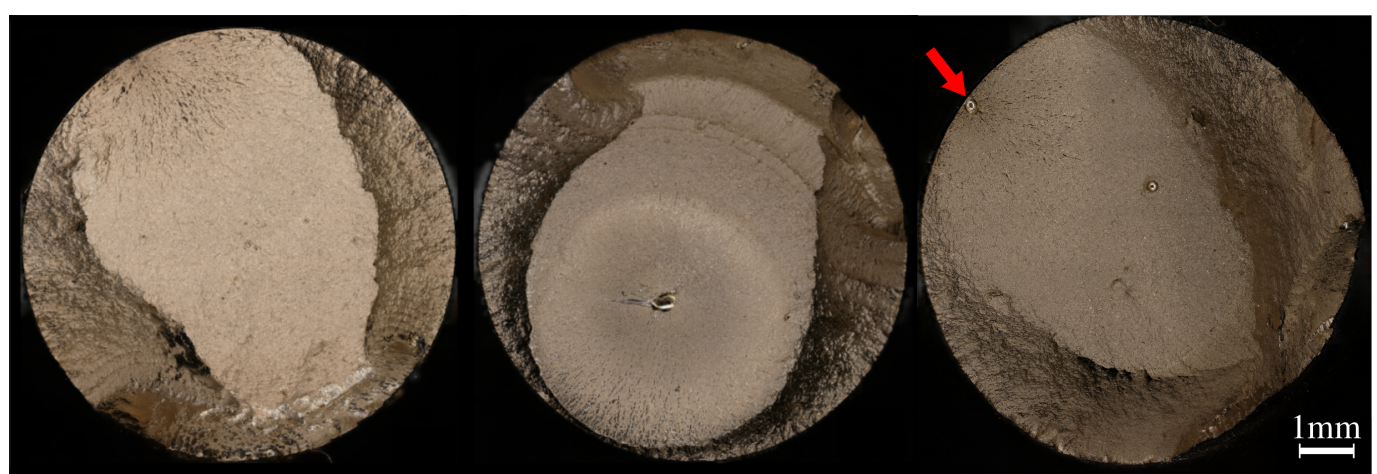

(a) (b)

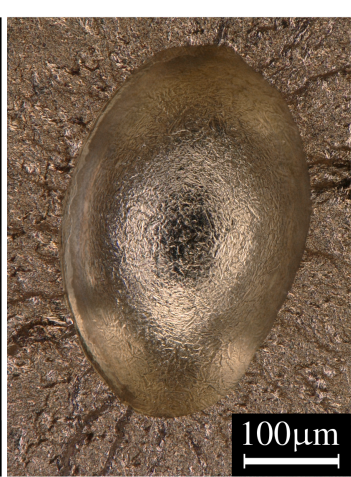

(d)

FIGURE 7 - Failed specimen fractographies: healthy sample (a), internal pore (b), with multiple pores including a sub-surface pore (c) and typical shape of a critical pore (d)

From the fractography analysis, we see that defects dimensions vary from $100 \mu \mathrm{m}$ to $1 \mathrm{~mm}$ and they are randomly placed, far or close to the edge, some even communicating with the outside of the sample (Figure 7). Most of the defects are bean-shaped or even spherical for some, with a very smooth surface (Figure 7d). This geometry is typical of gas pores. Other defects, such as lack of fusion, induce very intricate severe crack-like shapes and were not observed in the present material.

For all of the porous samples, a unique pore appears to have initiated cracks that actually led to failure. Multiple pores can be revealed but the discrepancy in criticality is such that there is no ambiguity as to which pore was responsible for the premature fatigue failure (Figure 7c). These defects will be referred to as critical pores.

Considering the pore population it appears that the fatigue samples cannot be considered as an RVE. The knowledge of the size and position of the defects in a sample is essential to predict fatigue life. The initial population of pores in a given volume must be characterized. Fatigue samples fractographies only give information on the most critical pores, that is not necessarily representative of the initial population. Tomography appears the best suited experimental method for void characterization, in addition of being a non-destructive technique. 


\subsection{Tomography}

Large volumes of metal cannot be directly analyzed by tomography. Cylindrical fragments of a maximum diameter of $16 \mathrm{~mm}$ were machined for a total analyzed volume of $45667 \mathrm{~mm}^{3}$. The tomography analyses were performed by CRT Morlaix using a Phoenix v|tome|xsv system. The scans were set at a voltage of $210 \mathrm{kV}$ and an intensity of $100 \mu \mathrm{A}$. Each scan is composed of 1800 images of $333 \mathrm{~ms}$ exposure time. A resolution of $21.26 \mu \mathrm{m}$ is achieved. Voids are detected using the VG Studio built-in tool and rendered in 3D (Figure 8). Parameters such as equivalent diameter, sphericity, projected surfaces and minimal distance to free surface are available.

The present material contains large pores, the mean diameter being $234 \mu \mathrm{m}$, with sizes up to $900 \mu \mathrm{m}$. The pores are considerably larger than microstructure grains. As expected from the metallography analysis and fatigue tests, the void density is very low, with $d=0.006 \mathrm{~mm}^{-3}$. The measured material density is actually greater than $99.999 \%$, showing that, despite a well parameterized process resulting in a dense material, isolated pores can considerably affect the fatigue results. Given the low pore density, a fatigue sample contains only a few defects of various sizes and positions, which is coherent with the experimental scatter. No cracks or lack of fusion-like geometries were observed in the analyzed volumes.

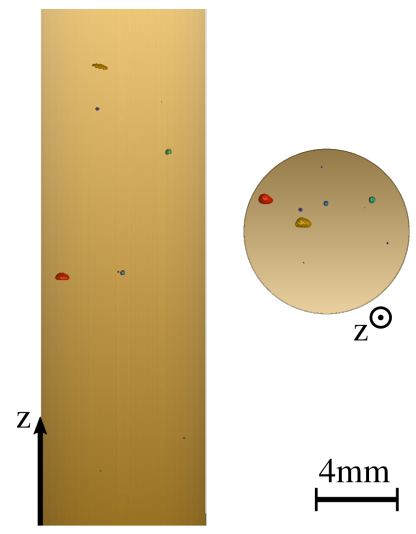

FIGURE 8 - Tomography void detection for an $8 \mathrm{~mm}$ diameter cylindrical specimen

No cluster of pores were observed in the fragments. The cause for the presence of pores is believed to be either the quality of the input wire [12,13], or the deposition parameters such as the intensity or voltage. Although, for the present blocks, there was no change in wire input and the deposition parameters were stable, given the simple geometry. As a result, a homogeneous random pore distribution is supposed.

The mean sphericity of all the detected pores (ratio between the void surface and the minimum enclosing ball surface) is equal to 0.62 (Figure 9b) and most of the pores have a regular and smooth shape. Assuming that the pores are spherical allows for a simple but still representative model. The equivalent diameter is determined by considering the area projected onto a plane perpendicular to the building direction of the block, as is done for fractography analysis (Figure 9c). It is worth to notice that the present WAAM defects are of different nature than those usually addressed in powder-based processes studies which have more intricate shapes, such as lack of fusion defects $[56,57]$.

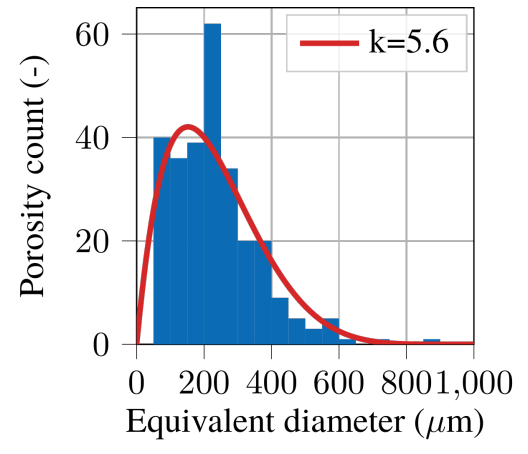

(a)

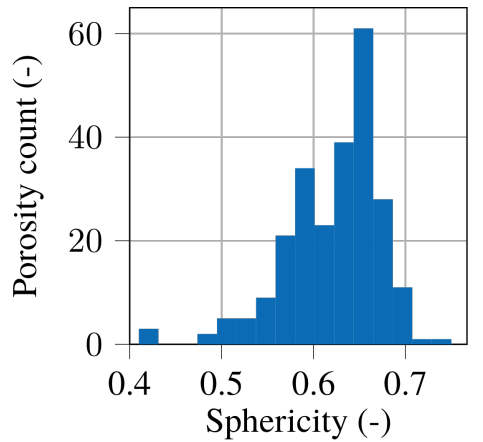

(b)

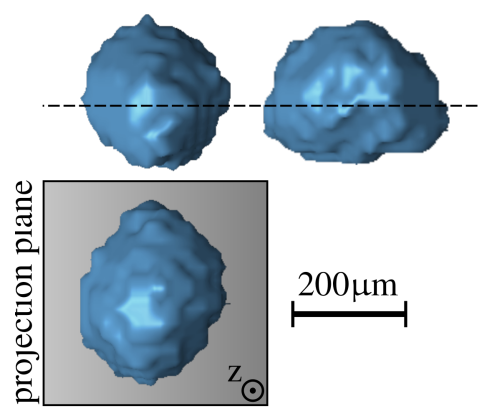

(c)

FIGURE 9 - Identification of parameter $k$ on diameter distribution (a), sphericity factor distribution of all detected voids (b) and typical 3D shape of a WAAM pore of a 0.6 sphericity with its projection plane for the determination of the equivalent diameter (c)

The pore population can then be described by a density parameter (number of voids per unit of volume) and a beta distribution on 
diameters, defined as

$$
n(D)=\frac{D\left(D_{\text {lim }}-D\right)^{k}}{D_{\text {lim }}^{k+1}} \frac{1}{w}
$$

where $n$ is the number of pores, $D$ the diameter, $D_{\text {lim }}$ the distribution upper limit, $k$ the intensity and $w$ a normalisation parameter. The two parameters of the beta law $D_{\text {lim }}$ and $k$ have opposite influence on the distribution: the higher the value of $k$, or the smaller $D_{\text {lim }}$ is, the smaller the diameters. The value of $D_{\text {lim }}$ is set at $1 \mathrm{~mm}$ in the present case, to ensure the existence of cases up to $1 \mathrm{~mm}$ in diameter, as the maximum experimental value is $900 \mu \mathrm{m}$. $k$ is identified on tomography results as illustrated on Figure $9 \mathrm{a}$.

Finally, the identified values of density and $k$ of the WAAM bronze-aluminium pore population are given in Table 3.

\begin{tabular}{|c|c|c|}
\hline$D_{\text {lim }}(\mu \mathrm{m})$ & Density $\left(\mathrm{mm}^{-3}\right)$ & $k(-)$ \\
\hline 1000 & 0.006 & 5.6 \\
\hline
\end{tabular}

TABLE 3 - Parameters of the initial population of pores

\subsection{Self-heating behaviour}

Fatigue properties of most metallic materials can be evaluated through self-heating tests. An empirical post-processing method allows for the determination of the fatigue strength [38]. In light of this, a self-heating approach is considered, in which the small number of pores per sample should not have any influence.

A specific experimental protocol is set up to characterize the self-heating behaviour of a material under cyclic sollicitation [41, 58]. A sample is subjected to successive cyclic loading blocks of increasing amplitude $\Sigma_{0}$ (Figure 10a). The sample and the grips temperature are measured using thermocouples. Since only the bulk sample mean temperature is considered, it is said to be a $0 \mathrm{D}$ approach. Under cyclic loading, the self-heating temperature $\theta$ is defined as

$$
\theta=T_{S}-\frac{T_{U G}+T_{L G}}{2}
$$

where $T_{S}, T_{U G}$ and $T_{L G}$ are the temperatures of the sample, the upper grip and the lower grip respectively. For each loading block, $\theta$ rises until reaching its steady-state temperature $\bar{\theta}$ (Figure 10b). The self-heating curve shows the evolution of $\bar{\theta}$ according to the stress amplitude $\Sigma_{0}$ (Figure 10c).

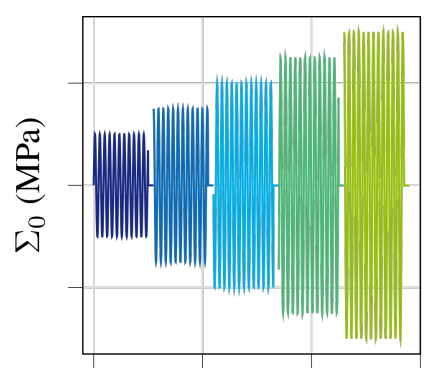

Time (s)

(a)

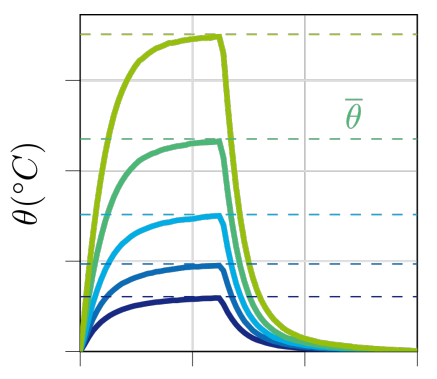

Time (s)

(b)

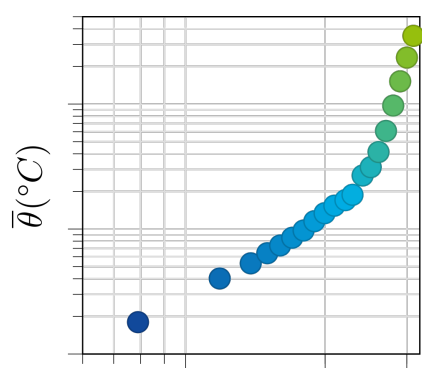

$\Sigma_{0}(\mathrm{MPa})$

(c)

FIGURE 10 - Illustration of the self-heating test protocol: cyclic loading blocks of constant amplitude are applied successively (a), corresponding self-heating temperature is measured using thermocouples (b) and the resulting self-heating curve plots the stabilized temperature $\bar{\theta}$ versus the loading amplitude $\Sigma_{0}$, here in log-log scale (c)

Self-heating tests were performed using a vibrophore with cylindrical samples of $8 \mathrm{~mm}$ in diameter and a $24 \mathrm{~mm}$ gauge length. Loading blocks of 30,000 cycles were applied at a frequency of $130 \mathrm{~Hz}$ at four different load ratios $R=\frac{\Sigma_{\min }}{\Sigma_{\max }}(-1,-0.6,-0.3$ and 0 ) to explore the influence of the mean stress on self-heating: $R=-1$ corresponds to a tension-compression test with zero mean stress, while $R=0$ corresponds to a pure tension test with equal mean stress and amplitude. Four samples were tested at the same load ratio $R=-1$ (samples $S 1$ to $S 4$ ). The results show good repeatability (Figure 11), as no significant scatter is observed for the four self-heating curves at $R=-1$. As for other loading ratios, the closer to $R=0$, the higher the mean stress, the more premature 
the self-heating. This premature self-heating of the material demonstrates the detrimental effect of a tensile mean stress on fatigue performances. This is a typical observation for metals [59], giving good confidence in the self-heating results.

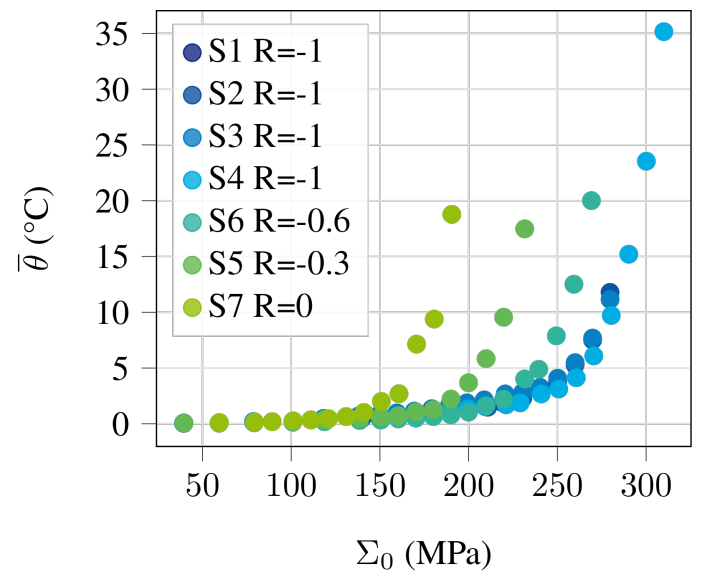

FIGURE 11 - Self-heating curves of seven WAAM samples at $R=-1, R=-0.6, R=-0.3$ and $R=0$ in linear scale

According to the tomography results, there are seven pores in each self-heating sample on average. Despite their strong impact on fatigue life, they have no influence on the dissipation behaviour of the material under cyclic loading, given the repeatability of the results. There are not enough defects in a sample to be detected by a OD approach, as opposed to the casting case [49]. Regarding self-heating behaviour, the tested samples do constitute an RVE, in contrast to fatigue. In light of this, we understand that self-heating tests allow for the characterization of the dense WAAM material. In other words, a virtually healthy WAAM material, with no pore, can be characterized through self-heating tests.

The self-heating behaviour under multiaxial stress [60] was also explored. Tubular samples 3mm thick and 44mm gauge length were extracted from the WAAM blocks (Figure 2c). The tests were performed on an MTS tension-torsion servo-hydraulic test rig of a capacity of $250 \mathrm{kN}$ at a frequency of $10 \mathrm{~Hz}$. The present material mechanical behaviour is time independent. In that context, the self-heating temperature is linear with respect to the loading frequency while the intrisic dissipation is independent [41]. The difference in frequency between multiaxial and uniaxial tests has no effect whatsoever on the identification of model parameters. The goal of the multiaxial self-heating test is to determine the surface of iso-self-heating in the $(\sigma ; \sqrt{3} \tau)$ Mises space given a self-heating temperature threshold $\theta_{t h}$. The multiaxial loading paths are proportional without mean stress. They are referred to by their angle to the pure tension-compression path in the Mises plane. A total of eight multiaxial stress paths were explored, from 0 to $\frac{7 \pi}{8}$. The equivalent paths $\frac{5 \pi}{8}$ and $\frac{7 \pi}{8}$ as well as $\frac{\pi}{8}$ and $\frac{3 \pi}{8}$ allow a potential history effect to be detected. To limit such an effect, consecutive stress paths should be orthogonal, giving the following exploration order: $0 \rightarrow \frac{4 \pi}{8} \rightarrow \frac{\pi}{8} \rightarrow \frac{5 \pi}{8} \rightarrow \frac{2 \pi}{8} \rightarrow \frac{6 \pi}{8} \rightarrow \frac{3 \pi}{8} \rightarrow \frac{7 \pi}{8}$. For each stress path, the regular self-heating test protocol is applied until the threshold $\theta_{t h}$ is reached, then the following stress path is explored.

The resulting iso-self-heating surface for the WAAM material is plotted in Figure 12 and compared to the Mises equivalent stress surface. As the stress field is not homogeneous, the results are plotted in shear stress amplitude averaged over the sample's section $\overline{\tau_{a}}$.

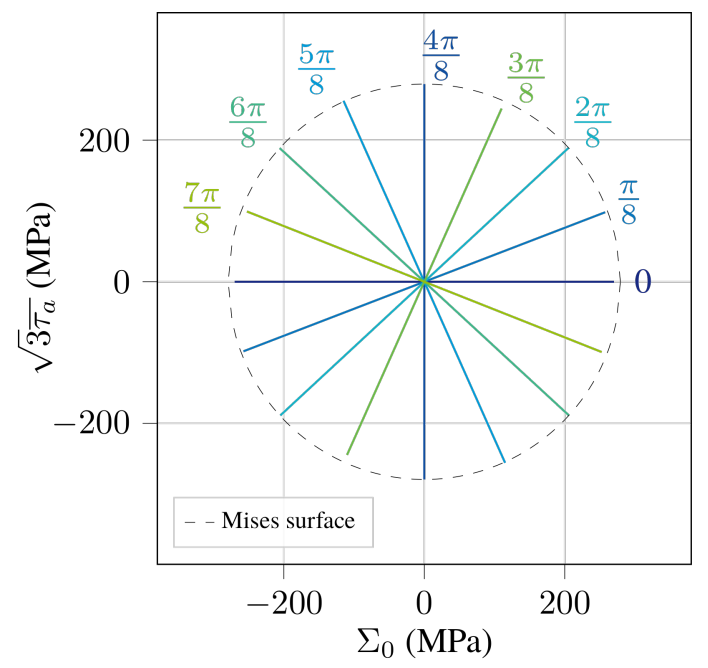

FIGURE 12 - Iso-self-heating test results for the threshold $\theta_{t h}=0,3^{\circ} \mathrm{C}$ compared to the Mises surface (in dotted line) 
The iso-self-heating surface corresponds to the Mises equivalent stress surface, with only a mean error of $2 \%$. This justifies the use of Mises stress as the equivalent stress amplitude in the case of a multiaxial loading.

Finally, it appears that given the low density of defects in the WAAM material, fatigue samples cannot possibly constitute a RVE. The pores have a first order influence on fatigue life and induce a scatter that makes conventional tests hard to interpret. On the other hand, self-heating tests are not influenced by WAAM pores, and the results show good repeatability. The material characterized by selfheating corresponds to a healthy WAAM material, with virtually no defects. In the next step, a specific model is used to describe the fatigue behaviour of this healthy material using its self-heating results. It will be used as the constitutive material of porous structures modeled by FEA in order to predict the influence of pores on fatigue life.

\section{A probabilistic fatigue model based on self-heating behaviour}

In order to link the self-heating behaviour to fatigue properties, the probabilistic model introduced in [41] is adopted. This model allows for the determination of the fatigue strength as well as the SNP curves from the self-heating experimental results.

It is important to bear in mind that in the present paragraph, no influence of any defect whatsoever is considered. The material characterized through self-heating is fully dense, and is referred to as a virtually healthy WAAM material.

\subsection{Probabilistic fatigue model}

The fatigue behaviour of the material is interpreted through a specific RVE, composed of one elasto-plastic matrix and one active plastic site (Figure 13a). The plastic behaviour of the site only differs from the matrix by its local elastic yield $\sigma_{y}^{\mu}$. If the equivalent

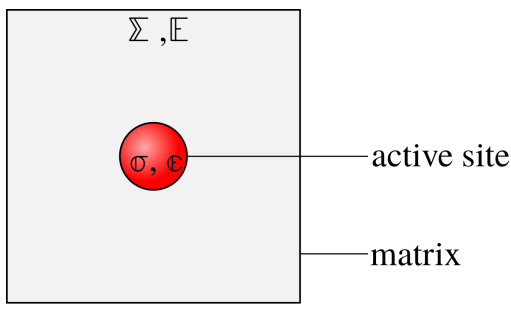

(a)

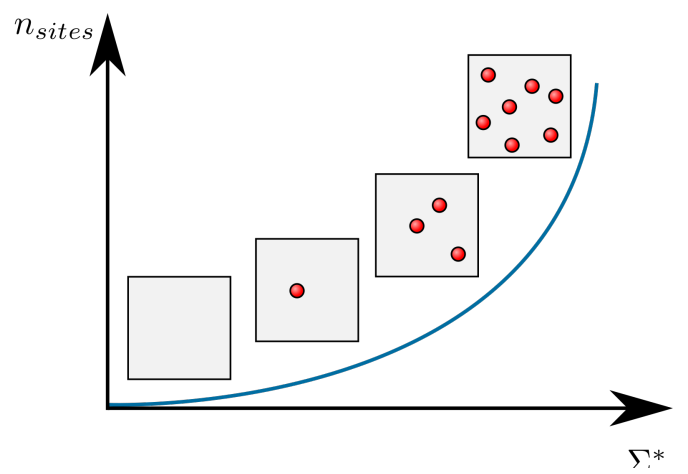

(b)

FIGURE 13 - RVE to describe the material's behaviour in fatigue (a) and the number of active sites against cyclic loading amplitude following a Poisson Point Process (b)

amplitude $\Sigma_{a}^{*}$ of a homogeneous multiaxial macroscopic cyclic loading exceeds the site local yield $\sigma_{y}^{\mu}$ then the site is activated. Considering $\sigma_{y}^{\mu}$ as a random variable, the number of active sites $n_{\text {sites }}$ in a volume $V$, under a macroscopic cyclic loading of amplitude $\Sigma_{a}^{*}$ (Figure 13b) is driven by a Poisson Point Process (PPP)

$$
n_{\text {sites }}(V)=\lambda V=\frac{1}{V_{0}}\left(\frac{\Sigma_{a}^{*}}{S_{0}-\alpha^{*} \frac{I_{1 m}}{3}}\right)^{m} V
$$

where $\lambda$ is the intensity of the point process, $I_{1_{m}}$ the mean value of the stress tensor's first invariant and $V_{0}, S_{0}, \alpha^{*}$ and $m$ the material parameters of the model. The choice of RVE and the PPP law is made from microstructural observations performed in [61]. In particular, active sites represent the persistent slip bands that appear under high amplitude cyclic loading. In other words, active sites bear witness to fatigue damage. The weakest link hypothesis is then proposed: as soon as one site is active, fatigue failure will ensue. From the PPP (Equation 3), the probability of having $k$ active sites in a volume $V$ is

$$
P_{k}(V)=\frac{(\lambda V)^{k}}{k !} e^{\lambda V}
$$


Then the probability of having at least one active site, or the failure probability $P_{F}$, is

$$
P_{F}=1-P_{k=0}=1-\exp \left[\frac{-V}{V_{0}}\left(\frac{\Sigma_{a}^{*}}{S_{0}-\alpha^{*} \frac{I_{m}}{3}}\right)^{m}\right] .
$$

This corresponds to the Weibull model [62], where $S_{0}$ is the scale parameter and $m$ the Weibull modulus: the higher $m$, the lower the intrinsic scatter of the material's fatigue data. Then, the mean fatigue strength $\bar{\Sigma}_{\infty}$ and its standard deviation $\overline{\bar{\Sigma}}_{\infty}$ according to the failure probability $P_{F}$ are defined as

$$
\left\{\begin{array}{l}
\bar{\Sigma}_{\infty}=\int_{0}^{\infty} \Sigma_{a}^{*} \frac{d P_{F}}{\mathrm{~d} \Sigma_{0}} \mathrm{~d} \Sigma_{0} \\
\overline{\bar{\Sigma}}_{\infty}=\int_{0}^{\infty}\left(\Sigma_{a}^{* 2}-\bar{\Sigma}_{\infty}^{2}\right) \frac{\mathrm{d} P_{F}}{\mathrm{~d} \Sigma_{0}} \mathrm{~d} \Sigma_{0}
\end{array}\right.
$$

or, using equation 5

$$
\left\{\begin{array}{l}
\bar{\Sigma}_{\infty}=\left(S_{0}-\alpha^{*} \frac{I_{1_{m}}}{3}\right)\left(\frac{V_{0}}{V}\right)^{1 / m} \Gamma\left(1+\frac{1}{m}\right), \\
\overline{\bar{\Sigma}}_{\infty}=\left(S_{0}-\alpha^{*} \frac{I_{1_{m}}}{3}\right)\left(\frac{V_{0}}{V}\right)^{1 / m} \sqrt{\Gamma\left(1+\frac{2}{m}\right)-\Gamma^{2}\left(1+\frac{1}{m}\right)},
\end{array}\right.
$$

where $\Gamma(x)=\int_{0}^{\infty} t^{x-1} \exp (-t) \mathrm{d} t$ is Euler's gamma function.

The finite life fatigue domain (i.e. for $\Sigma_{a}^{*} \geq \bar{\Sigma}_{\infty}$ ) can be described by the model when introducing an energy criterion. The dissipated energy is cumulated after each cycle [40], and the existence of a critical dissipated energy $E_{c}$ is assumed [60,63]

$$
E_{c}\left(P_{F}\right)=N_{f} \bar{\Delta}_{\text {site }}\left(P_{F}\right) V_{R V E}
$$

where $P_{F}$ is the considered probability of failure, $N_{f}$ the associated number of cycles to failure, $\bar{\Delta}_{\text {site }}$ the dissipation of one site (constant per cycle) and $V_{R V E}$ the volume of the RVE. In the case of this study, the energy criterion as proposed in [49] is used

$$
N_{R}\left(P_{F}\right)=\frac{A}{\left\langle\Sigma_{a}^{*}-\Sigma_{\infty}\left(P_{F}\right)\right\rangle^{2}}
$$

where $A$ is a material parameter depending on $E_{c}$, with $\langle X\rangle=X$ if $X>0$ and $\langle X\rangle=0$ otherwise. The value of $A$ is determined from at least one experimental fatigue failure value. The fatigue strength $\bar{\Sigma}_{\infty}\left(P_{F}\right)$ is evaluated using

$$
\frac{\log \left(1-P_{F}\right)}{\log (1-0.5)}=\left(\frac{\Sigma_{\infty}\left(P_{F}\right)}{\Sigma_{\infty}(0.5)}\right)^{m}
$$

where it is assumed that $\Sigma_{\infty}(0.5)=\bar{\Sigma}_{\infty} \frac{\ln (2)^{1 / m}}{\Gamma(1+1 / m)} \approx \bar{\Sigma}_{\infty}$ (the greater the value of $m$, the more accurate the approximation).

With the proposed fatigue model, the whole cluster of Stress, Number of cycles and Probability (SNP) curves can be plotted using equation 9 with the material parameters $S_{0}, V_{0}, \alpha^{*}$ and $m$. These parameters are identified from the self-heating tests results (Table 4).

The microplastic sites described in the RVE are not to be confused with the WAAM-induced pores. The void density is too low to have any influence on the self-heating results, as seen in the experimental results (Figure 11). While a methodology was proposed for casting in which active sites were assimilated to defects [49], it is not possible for WAAM.

\subsection{Model identification}

Self-heating results allow for the identification of the model's parameters by relating the RVE dissipation under cyclic loading to the self-heating temperature. From the heat equation integrated over one cycle $N$, and considering assumptions relative to the context of a self-heating test, we have [40]

$$
\dot{\theta}^{0 D}(N)+\frac{\theta^{0 D}(N)}{\tau_{e q}^{0 D}}=f_{r} \frac{\Delta^{0 D}(N)}{\rho c},
$$

with $\tau_{e q}^{0 D}=\left[\frac{1}{\rho c}\left(\frac{2 h_{\text {grip }}}{L}+\frac{2 h_{a i r}(e+b)}{e b}\right)\right]^{-1}$ the characteristic time of thermal equilibrium $\left(h_{g r i p}\right.$ and $h_{a i r}$ the heat exchange coefficient on the grips and the air, respectively), $f_{r}$ the loading frequency, $\rho$ the density, $c$ the heat capacity and $\Delta^{0 D}(N)$ the intrinsic dissipation of the sample over one cycle. Experience shows that, on the studied material, the self-heating stabilises after a few cycles (Figure 10b) 
meaning that $\Delta^{0 D}(N)$ is steady over one cycle. We can write

$$
\bar{\theta}^{0 D}=\tau_{e q}^{0 D} f_{r} \frac{\bar{\Delta}^{0 D}}{\rho c} .
$$

From equation 12, we understand that a self-heating curve is a direct image of the intrinsic dissipation $\bar{\Delta}^{0 D}$ of the material under cyclic loading. The expression of this dissipation is detailed using the material's RVE composed of an elastoplastic matrix and active microplastic sites (Figure 13a), here under uniaxial loading of an amplitude $\Sigma_{0}$

$$
\bar{\Delta}^{0 D}=\frac{\rho c}{f_{r} \tau_{e q}^{0 D}}\left(\alpha \Sigma_{0}^{2}+\delta \Sigma_{0}^{m+2}\right)
$$

where

$$
\left\{\begin{array}{l}
\alpha=f_{r} \tau_{e q}^{0 D} K_{1}, \\
\delta=f_{r} \tau_{e q}^{0 D} K_{2} \frac{V}{V_{0}\left(S_{0}-\alpha^{*} \frac{I_{1 m}}{3}\right)^{m}} \frac{1}{(m+1)+(m+2)},
\end{array}\right.
$$

where $K_{1}$ and $K_{2}$ are the hardening parameters of the matrix and the sites [41]. The term with factor $\alpha$ in equation 13 corresponds to the dissipation of the matrix while the term with factor $\delta$ represents the dissipation of the active sites. These two dissipation mechanisms can be observed in practice on the self-heating curve in logarithmic scale (Figure 10c) where two distinct slopes appear. These are addressed as the first regime (matrix dissipation) and the second regime (matrix and active sites dissipation). The parameter $\alpha$ is identified on the first half of the curve by imposing a slope of 2 (Figure 14a). Then $\delta$ and $m$ are determined by interpolation of the last values of the curve from which the matrix contribution is withdrawn (Figure 14b) as such

$$
\bar{\theta}_{\text {second }}\left(\Sigma_{0}\right)=\bar{\theta}\left(\Sigma_{0}\right)-\alpha \Sigma_{0}^{2}
$$

The value of $\alpha^{*}$ (Equation 3) is identified from multiple self-heating tests at different stress ratios. $S_{0}$ is determined from $\delta$ by imposing $V_{0}$ equal to the volume of the self-heating sample's reduced section. The identified model gives a very good description of the selfheating curve (Figure 14c). In practice, the last load step of a self-heating test is extended until failure of the specimen to allow for the identification of parameter $A$. It must be noted that, as we intend to characterize the healthy WAAM material, only healthy fatigue failures (without pores in the failed section) must be considered to determine $A$.

\begin{tabular}{|c|c|c|c|c|}
\hline$V_{0}\left(\mathrm{~mm}^{3}\right)$ & $S_{0}(\mathrm{MPa})$ & $m(-)$ & $\alpha^{*}(-)$ & $A\left(\mathrm{MPa}^{2}\right)$ \\
\hline 1206 & 250 & 14 & 1.6 & $1.92 \times 10^{9}$ \\
\hline
\end{tabular}

TABLE 4 - Material parameters of the probabilistic model

The first regime is related to matrix dissipation only, while the second regime corresponds to the activation of dissipative microplastic sites. The transition from one regime to another marks the activation of at least one site. According to the weakest link hypothesis, the corresponding stress threshold is assimilated to the fatigue strength of the material. In practice it is identified as the smallest stress amplitude not dominated by the first regime (Figure 14c).

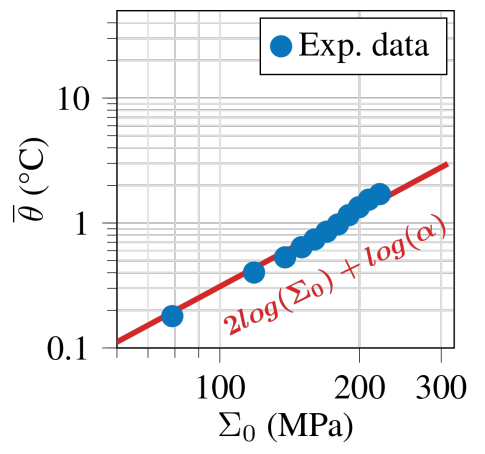

(a)

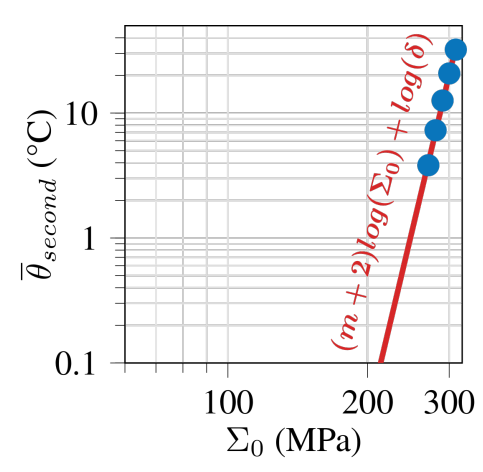

(b)

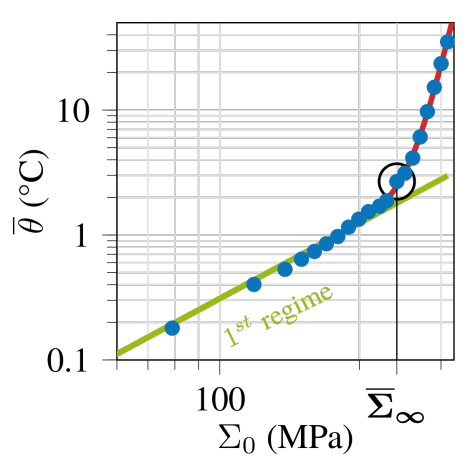

(c)

FIGURE 14 - Identification of the first regime (a), the second regime (b) and the fatigue strength at $R=-1$ (c)

Finally, self-heating tests, with the associated fatigue model, allow for the fatigue behaviour of the healthy WAAM material to be 
fully determined. The probabilistic model describes the intrinsic fatigue scatter of the material. The scatter resulting from pores is not covered by the model as presented. By considering the porous fatigue sample as a structure with the healthy WAAM bronze as constitutive material, the influence of pores on fatigue is explored.

\section{Numerical fatigue campaign}

In this section, a statistical consideration of the influence of pores on fatigue is proposed through a numerical fatigue test campaign. The objective is to generate numerous porous samples and to compute their SNP curves from FE calculations, coupled with the probabilistic model. The resulting numerical fatigue data should display a scatter representative of the experimental scatter.

\subsection{Probabilistic model in the case of a porous sample}

The failure probability as expressed in equation 5 is valid for a volume $V$ submitted to a multiaxial homogeneous stress field of amplitude $\Sigma_{a}^{*}$ and mean stress $I_{1_{m}}$. This is the case of the healthy fatigue samples. The presence of a pore introduces multiaxial stress heterogeneity (Figure 15), whose intensity and spread must be considered. The intensity is parametrized with the stress concentration factor $K_{t}$ and the spread with an effective volume $V_{\text {eff }}$ [40], both defined as

$$
\left\{\begin{array}{l}
K_{t}=\frac{\Sigma_{a_{\max }}^{*}}{\Sigma_{a_{\text {mom }}}^{*}} \\
V_{\text {eff }}=H_{m} V \\
H_{m}=\frac{1}{V} \int_{V}\left[\frac{\Sigma_{a}^{*}(M)}{\Sigma_{a_{\max }}^{*}}\left(\frac{S_{0}-\alpha^{*} \frac{I_{1 m_{\max }}}{3}}{S_{0}-\alpha^{*} \frac{I_{1 m}(M)}{3}}\right)\right]^{m} d V
\end{array}\right.
$$

where $\Sigma^{*}$ is the Mises equivalent stress (chosen from the multiaxial self heating test), $\Sigma_{n o m}^{*}$ the nominal stress ( $\Sigma_{0}$ int he case of a healthy sample), $I_{1 m}$ the mean first invariant and $H_{m}$ the heterogeneity factor. The failure probability in the case of a structure with a heterogeneous stress field is then

$$
P_{F}=1-\exp \left[\frac{-V_{\text {eff }}}{V_{0}}\left(\frac{K_{t} \Sigma_{a_{n o m}}^{*}}{S_{0}-\alpha^{*} K_{t} \frac{I_{1 m_{n o m}}}{3}}\right)^{m}\right] .
$$

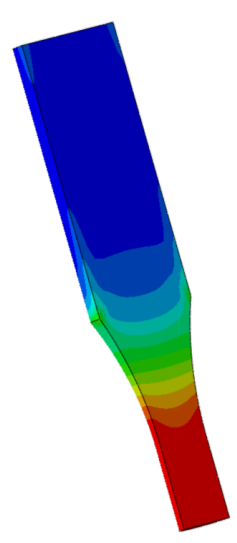

(a)

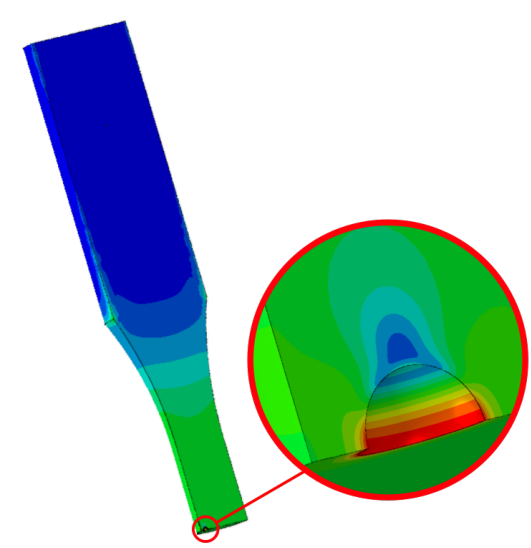

(b)

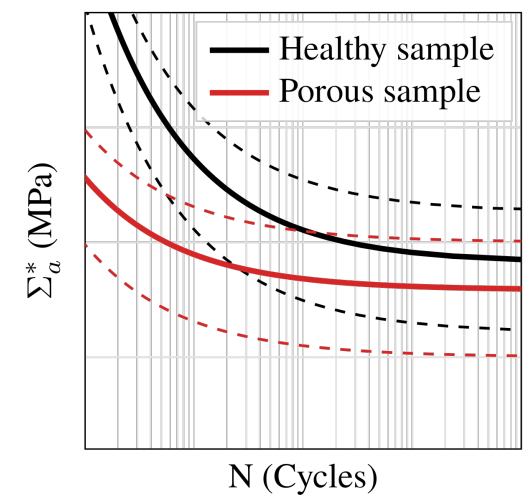

(c)

FIGURE 15 - Homogeneous stress field in a healthy sample (a), heterogeneity in a porous sample (b) and corresponding SNP curves (c)

The effective volume $V_{\text {eff }}$ stands for a theoretical volume subjected to the homogeneous stress field $K_{t} \underline{\underline{\Sigma}}_{n o m}$ that has the same failure probability as the original heterogeneous structure. In that sense, the model takes into account, not only the stress concentration introduced by the pore, but also the size of the concentration: the larger the zone of high stress, the more active micro plastic sites. From equations 9 and 10, the fatigue life of the structure for a failure probability $P_{u}$ is

$$
N_{R}\left(P_{u}\right)=\frac{A}{\left(\left\langle 1-\frac{\log \left(1-P_{u}\right)}{\log \left(1-P_{F}\right)}\right\rangle K_{t} \Sigma_{a_{n o m}}^{*}\right)^{2}} .
$$


From the knowledge of the stress state in a structure, the model gives the expression of its SNP curves. In this case, an elastic FE calculation of a porous sample allows for the computation of its fatigue performance for any nominal stress (Figure 15).

In what follows, no mean stress is considered, meaning $I_{1 m}=0$ and the stress ratio $R=-1$.

\subsection{Numerical samples}

In order to perform a numerical fatigue campaign, porous samples representative of the WAAM material must be generated. It is reminded that an idealized pore population was identified in the tomography results, with two parameters: the intensity of the diameter distribution law $k=5.6$ and the pore density $d=0.006 \mathrm{~mm}^{-3}$.

Numerical porous samples were generated through a six step process, as illustrated in Figure 16:

1. An initial plain volume $V$ is created.

2. A population of $n=V d$ pores is generated according to a beta law of intensity $k$ (equation 2).

3. The $n$ pores are randomly placed in the volume $V$. No inter-penetration of pores is allowed. If any is detected then one of the pores is removed and placed randomly until there is no inter-penetration.

4. $n_{s}$ sample gauge lengths are generated inside the initial volume $V$. The minimum distance between each sample is greater than the maximum void diameter $D_{\text {lim }}$ to avoid any shared pore ambiguity.

5. pores that intersects with sample's surface are attributed to the corresponding sample. This step illustrates the actual extraction of samples from a WAAM block.

6. Finally, for each sample, the pore cases are recorded and described by their diameter $D$ and distance to the edge $d_{e}$.

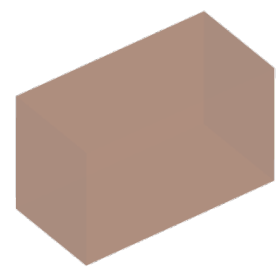

Creation of an initial volume $\mathrm{V}$

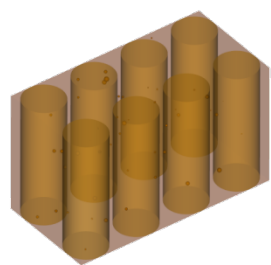

Subdivision in sample gage lengths

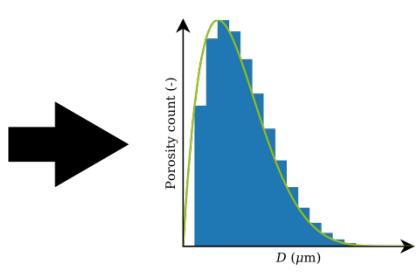

Generation of diameters from tomography results $(\mathrm{k} ; \mathrm{d})$

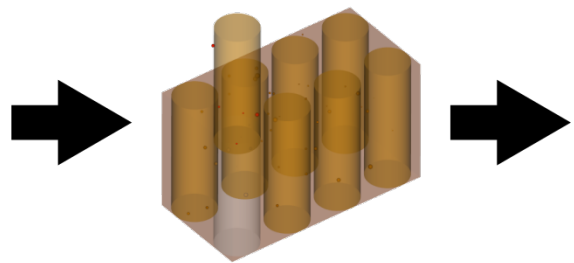

Extraction of the subvolumes

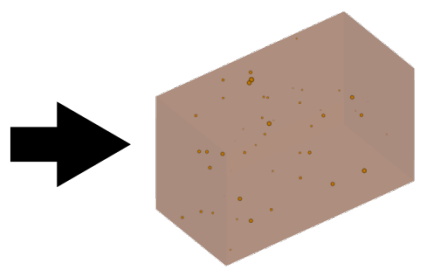

Random positioning of porosities without collisions

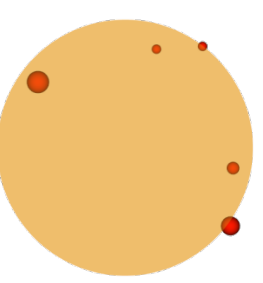

Detection of the porosity cases $\left(D ; d_{c}\right)$ for every sample

FIGURE 16 - Creation of a porous sample population according to tomography results

Given the random nature of the position of the pores, each porous sample generated is unique. Moreover, we want to be able to measure the influence of the initial pore population on fatigue by exploring values of $k$ and $d$. For this reason, an FE computation of the stress field for each sample seems unrealistic. Instead, the creation of a database of pore cases, ranging over $\left(D ; d_{e}\right)$, is proposed. Such a database is valid for any pore population $(k ; d)$ and only depends on mechanical parameters (namely the Poisson coefficient $\nu$, the Young modulus $E$ and the self-heating parameters $S_{0}, V_{0}$ and $m$ ).

\subsection{Pore case database}

In order to create a relevant pore case database, the influence of $\left(D ; d_{e}\right)$ on fatigue criticality must be explored.

Based on the tomography and fractography results, pores are considered as spherical voids. In this context, a pore is fully characterized by its diameter $D$ and its distance to the edge of the sample $d_{e}$ (centre of pore to the edge of sample, Figure 17b). Let us consider 30 pore cases of different diameters $D$ and distances to surface $d_{e}$, and compute their criticality level from the FEA stress 
fields, defined as the fatigue strength knock-down ratio $k_{d}$ for a $50 \%$ failure probability at $2.10^{7}$ cycles

$$
k_{d}=\frac{\sum_{\infty}^{\text {healthy }}\left(P_{F}=0.5\right)}{\sum_{\infty}^{\text {porous }}\left(P_{F}=0.5\right)} .
$$

To facilitate the comparison between pores of different sizes, the ratio $\frac{d_{e}}{D}$ will be considered instead of simply $d_{e}$. Values of $\frac{d_{e}}{D}$ above 0.5 stand for internal pores, while values below 0.5 stand for external ones. The case of a healthy sample can be represented by the virtual value $\frac{d_{e}}{D}=-0.5$, below which the pore would be outside of the sample. The results of the FEA computation for each case are shown in Figure 17a, as well as a schematic representation of the 30 pore cases on a same cross-section, with a criticality level according to $k_{d}$ (Figure $17 \mathrm{~b}$ ) and $K_{t}$ (Figure 17c).

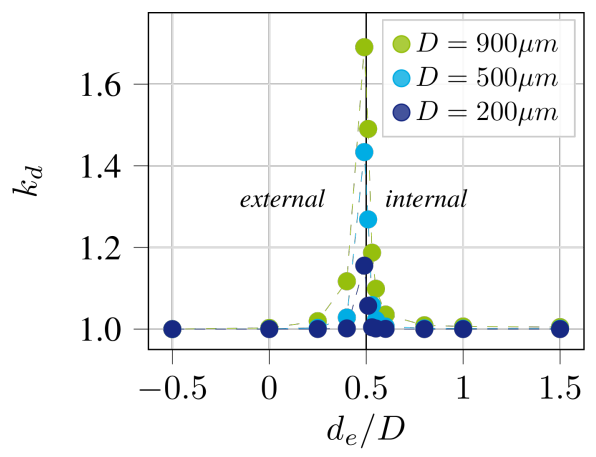

(a)

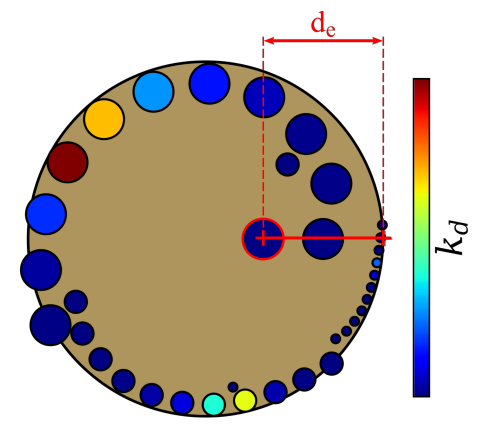

(b)

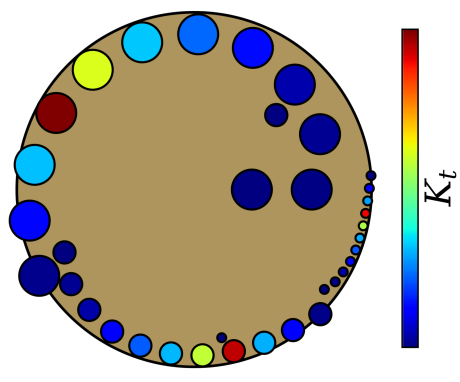

(c)

FIgURE 17 - Criticality for various $d_{e}$ and $D$ (a) and a representation of the 30 cases on a same cross-section, coloured according to their $k_{d}$ value (b) and $K_{t}$ value (c)

It appears that the knock-down ratio in fatigue strength $k_{d}$ is very sensitive to $d_{e}$, with an asymptotic like behaviour close to $\frac{d_{e}}{D}=0.5$ which marks the transition between internal and external pores. For values of $\frac{d_{e}}{D}$ above 1 , there is very little variation of $k_{d}$; cases with $\frac{d_{e}}{D}<1$ are then referenced as sub-surface defects.

The stress concentration factor $K_{t}$ would show a similar tendency as $k_{d}$ [22], although it would be unsensitive to the diameter $D$. This is because the effective volume $V_{e f f}$, used in the determination of $k_{d}$, is dependent on $D$. This is especially important when it comes to the criticality ranking of the pores. Figure 17 shows that in the sense of $K_{t}$, small sub-surface defects are as critical as bigger sub-surface defects. But in the sense of $k_{d}$, using the probabilistic model, small sub-surface defects appear to be less critical compared to bigger defects. While the stress intensities might be comparable, their spread, and therefore the $V_{e f f}$ values, are not. This model proposes an alternative to Murakami-like approaches [19] to take into account the influence of both the size and position of a defect on fatigue performances.

It appears that the distance to the surface is critical with regard to fatigue life, as it was observed for other AM materials [21,22]. From these observations, we understand that a fine discretization of $d_{e}$ around 0.5 is needed in the pore database, while values above 1.5 can be considered quasi-constant. The database used in this study comprises of 117 cases defined by the values detailed in Table 5. For each $\left(D ; d_{e}\right)$ from the database, the corresponding couple of values $\left(K_{t} ; V_{e f f}\right)$ is computed by FEA. Indeed, the probability of failure $P_{F}$ definition (equation 17) implies that the SNP curves of any pore case are fully determined by its $V_{e f f}$ and its $K_{t}$. The values $\left(K_{t}^{i} ; V_{e f f}^{i}\right)$ for any generated porous sample are then interpolated from the database values.

The evaluation of $V_{\text {eff }}$ involves the integration of the stress field at the exponent $m$, whose value is usually between 10 and 20 . This requires a precise computation of the stress heterogeneity around the pore. This can be achieved through structural zooming in the FEA model (Figure 18). Hexaedron quadratic elements were used, with a denser mesh close to the pore. Care was taken to obtain a converged stress field while keeping a reasonable computation time. Each pore case was effectively modeled: as the diameter of the pores is variable, while the sample's radius remains constant, no calculations are redundant.

The stress field away from the pore is homogeneous and uniaxial, the effective volume in this case is expressed

$$
V_{e f f}^{h o m}=\int_{V_{h o m}}\left(\frac{\Sigma_{a_{\text {nom }}}^{*}}{\sum_{a_{\text {max }}}^{*}}\right)^{m} d V=\left(V_{\text {sample }}-V_{\text {zoom }}-V_{\text {poro }}\right)\left(\frac{1}{K_{t}}\right)^{m},
$$

where $V_{\text {sample }}$ is the volume of the sample in its reduced section, $V_{z o o m}$ the volume of the structural zooming and $V_{\text {poro }}$ the volume 


\begin{tabular}{|c|c|}
\hline Diameter $D(\mu \mathrm{m})$ & Distance to surface $\frac{d_{e}}{D}(-)$ \\
\hline \hline 100 & 0 \\
200 & 0.25 \\
300 & 0.3 \\
400 & 0.4 \\
500 & 0.49 \\
600 & 0.51 \\
700 & 0.53 \\
800 & 0.55 \\
900 & 0.6 \\
& 0.8 \\
& 1 \\
& 1.5 \\
& 3 \\
\hline
\end{tabular}

TABLE 5 - Database discretization of $\left(D ; d_{e}\right)$

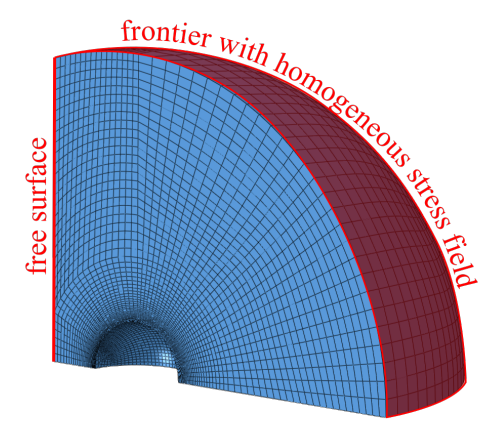

Figure 18 - FE structural zoom of a pore

of the pore (Figure 19). Then the effective volume of a sample with an unique pore $V_{e f f}^{i}$ is written

$$
V_{e f f}^{i}=\int_{V_{i}}\left(\frac{\Sigma_{a}^{*}(M)}{\sum_{a_{n o m}}^{*}} \frac{1}{K_{t}^{i}}\right)^{m} d V=V_{e f f}^{z o o m}+V_{e f f}^{h o m}
$$

where $K_{t}^{i}$ is the pore's stress concentration factor and $V_{e f f}^{z o o m}$ is the effective volume computed from the FEA of the structural zooming.

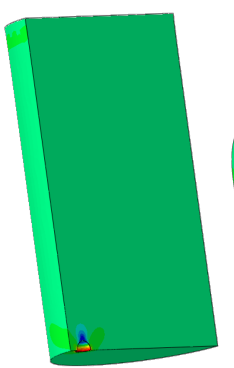

$V_{o f f}^{t o t}$

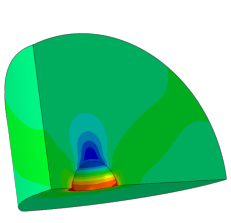

$V_{\rho f f}^{z o o m}$

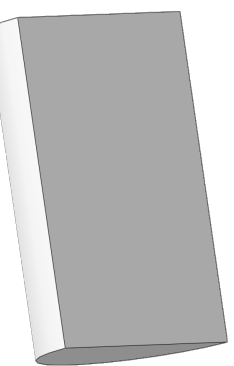

$V_{\text {sample }}$

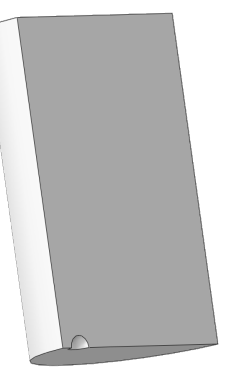

$V_{t o t}$

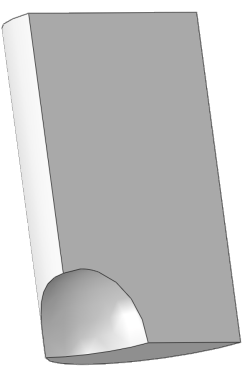

$V_{\text {hom }}$

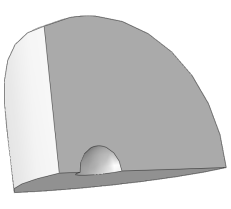

$V_{\text {zoom }}$

$V_{\text {poro }}$

FIGURE 19 - Representation of the different volumes at stake in the expression of the total effective volume of a porous sample

Despite the fact that the database is composed of samples with a single pore, it can be used to address the case of samples with multiple defects. Given the low defect density $\left(d=0.006 \mathrm{~mm}^{-3}\right)$, we assume that there is not any interaction between the pores stress field. Each pore can then be considered independently: its effective volume $V_{e f f}^{i}$ is interpolated from the database values as if it was the sample's sole defect. To be used in the context of multiple defects, this effective volume is normalized using the global maximum stress concentration factor $K_{t}^{\max }$. The effective volume of the homogeneous stress field away from the pore $V_{\text {sample }}\left(\frac{1}{K_{t}^{\text {max }}}\right)^{m}$ is 
redundant in each $V_{e f f}^{i}$ and so is removed. Finally, the total effective volume of a sample with $n_{\text {poro }}$ pores is written

$$
V_{e f f}^{\text {total }}=\sum_{i}^{n_{\text {poro }}} V_{e f f}^{i}\left(\frac{K_{t}^{i}}{K_{t}^{\text {max }}}\right)^{m}-\left(n_{\text {poro }}-1\right) V_{\text {sample }}\left(\frac{1}{K_{t}^{\text {max }}}\right)^{m}
$$

\subsection{Numerical SNP curve bundle}

A total of 100 porous samples are generated using the presented methodology (Figure 16). For every pore, $K_{t}^{i}$ and $V_{e f f}^{i}$ are interpolated from the database values. Finally, using Equations 17, 18 and 22, the SNP curves of each sample (Figure 20a), as well as a generalized SNP curve (Figure 20b) are plotted.

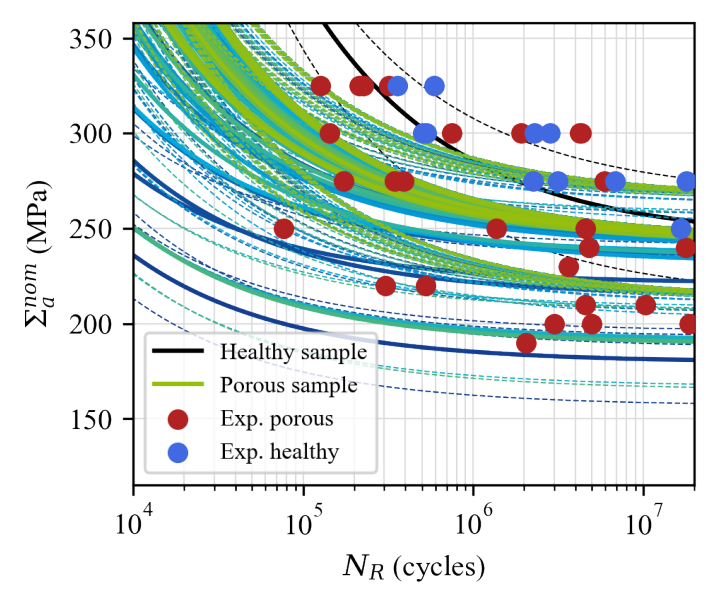

(a)

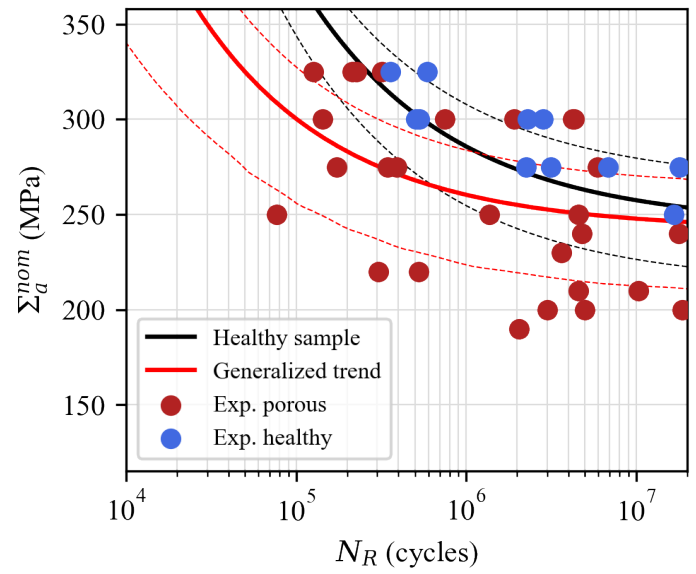

(b)

FIGURE 20 - SNP curves of the draw of 100 porous samples (a) and generalized trend (b)

First it can be noticed that the predicted healthy SN curve (in black on Figure 20) agrees closely with the better fatigue levels which correspond mostly to surface failures (blue dots) without pores. This increases confidence in the ability of the model to describe the behaviour of the healthy material with virtually no defects.

As for the porous specimen, the numerical fatigue scatter is representative of the experimental data. A few SNP curves show very premature fatigue failure (lowest curves in Figure 20a), which correspond to large sub-surface defects. Given the value of $k=5.6$, diameters tend to be concentrated around smaller values from $100 \mu \mathrm{m}$ to $300 \mu \mathrm{m}$, while diameters above $500 \mu \mathrm{m}$ are rare (Figure $9 \mathrm{a}$ ). This explains the small amount of very low fatigue life curves and their limited impact on the generalized SNP curve. Density $d$ is also responsible for these especially critical defects: if there are more pores in the initial extraction volume, it is more likely to find sub-surface defects in the samples.

Finally, the presented model takes into account both the intrinsic scatter of the virtual healthy material and the scatter induced by WAAM pores. The first is identified by self-heating tests using the probabilistic model. The second is determined through the statistical generation of porous structures, based on the knowledge of the real initial pore population observed by tomography.

The parameterization and identification of the real defect population appear essential to the fatigue dimensioning of such material. Parameters $k$ and $d$ act as a novel set of material parameters. To validate even further the presented methodology, tomography results are compared with the model's prediction, based on only the knowledge of fractography observations.

\subsection{Critical pore population}

The critical pore population is determined experimentally using the fractography results. It is characterized by a mean equivalent diameter $\bar{D}_{\text {crit }}$ and a mean distance to the edge ratio $\frac{\bar{d}_{e}}{D_{c r i t}}$. The critical population is only an image of the initial population as seen through the prism of fatigue. Both populations must not be confused. The present probabilistic model can generate a numerical critical pore population given values of $k$ and $d$, of the initial pore population. In the sense of the model, the critical pore of a sample is the one with the highest knock-down ratio $k_{d}$, when considered independently from the other pores.

Generating 10,000 draws of 100 samples with values of $(k ; d)$ ranging over $([1: 10] ;[0.0008: 0.008])$, the diameter $D_{\text {crit }}$ and the distance to the edge $\frac{d_{e}}{D}$ crit of critical defects are recorded. The influence of $k$ and $d$ of the initial population on $\bar{D}_{c r i t}$ and $\frac{\bar{d}_{e}}{D}$ crit 
of the critical population is plotted in Figure 21. As could be expected, the influence of $k$ on the mean critical diameter $\bar{D}_{c r i t}$ is more important than that of $d$. On the other hand the density $d$ has a strong influence on the mean distance to the edge ratio $\frac{\overline{d_{e}}}{D}$ crit $:$ the more pores, the more chances for sub-surface defects.

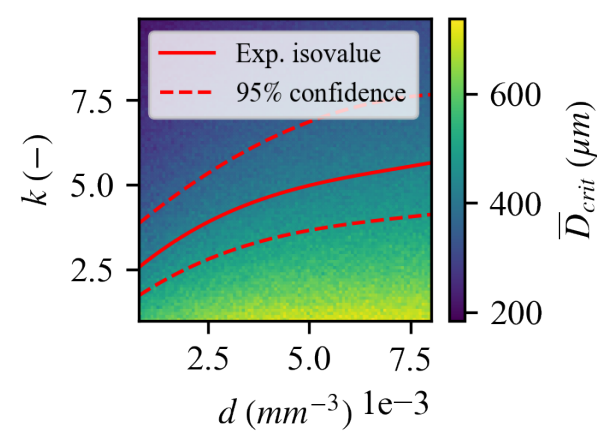

(a)

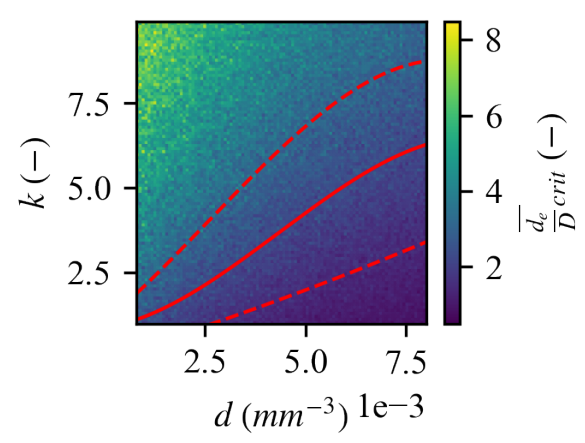

(b)

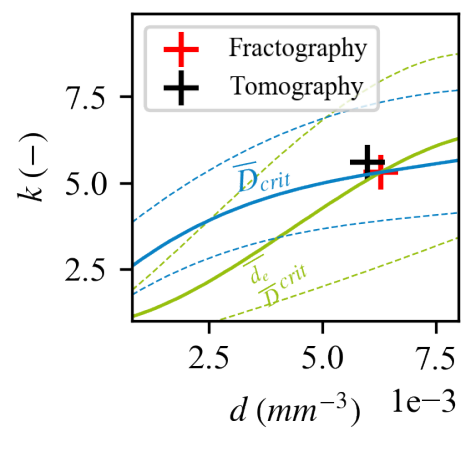

(c)

FIGURE 21 - Influence of the initial pore population's parameters $k$ and $d$ on the critical pore parameters $\bar{D}_{\text {crit }}$ (a) and $\frac{\bar{d}_{e}}{D}$ crit (b) with experimental iso-values, and comparison of the identification of $(k ; d)$ from fractographies and from tomography (c)

The critical pore population's parameters were determined experimentally on the basis of the fractography of 24 porous fatigue samples. The corresponding isovalues are plotted on the parameter maps in Figure 21 as well as their 95\% confidence interval (red lines). The intersection of the two isovalue curves should provide for the initial population $(k ; d)$ that induced the experimental critical population $\left(\bar{D}_{\text {crit }} ; \frac{\bar{d}_{e}}{D_{\text {crit }}}\right)$ according to the model (Figure $21 \mathrm{c}$ ). On the other hand, the initial population was identified experimentally by tomography. Both populations are represented in Figure 21c by their corresponding $k$ and $d$ values (one from the tomography analysis: black cross, and one from the model: red cross). The initial population parameters identified using the model are in very good accordance with the tomography results. This validates the capacity of the model to determine the most critical defect among multiple candidates. This increases confidence in the pore criticality ranking of the model, through the use of the stress concentration $K_{t}$ and the effective volume $V_{e f f}$.

\section{Summary and conclusions}

The effect of process-induced pores on the fatigue performance at $R=-1$ of bronze-aluminium machined WAAM samples was investigated in this study. On the basis of the experimental results, the following observations were made:

1. The WAAM material's microstructure is affected by the process. Heterogeneities in grain size highlight the frontiers between the different weld beads. Care must be taken with the dimensions of the samples to study an RVE in the sense of the microstructure.

2. The quasi-static mechanical properties in tension along the building direction of the heat treated WAAM material show considerably better strength and elongation before failure than its as-cast counterpart.

3. Conventional tension-compression fatigue tests results $(R=-1)$ show significant scatter, caused by the presence of large isolated pores. The samples do not constitute an RVE in the sense of fatigue behaviour.

4. Despite the important scatter in fatigue, the machined WAAM material shows better overall fatigue performance than its as-cast counterpart.

5. Tomography analysis allows for the identification of an idealized population through two parameters: the beta distribution intensity $k$ and the pore density $d$. Despite the low density $(<0.001 \%)$, the pores have a critical influence on fatigue life.

6. The self-heating behaviour is not affected by the presence of these rare pores. The samples do constitute a RVE in the sense of self-heating. The measured dissipation is equivalent to that of a dense sample with no pores, referred to as a virtually healthy material.

Through the set-up of a numerical methodology based on a probabilistic fatigue model, the following conclusions can be drawn:

1. A probabilistic model allows for the prediction of fatigue properties from self-heating measurements. The fatigue behaviour of the virtually healthy material is computed and used as reference. Self-heating tests and the associated model appears to be a good alternative to obtain fatigue properties for WAAM materials, as conventional tests are polluted by inevitable scatter. 
2. The probabilistic model is used on the porous sample structure and can rank pores according to their criticality. The criticality of a pore is defined as the knock-down ratio in fatigue strength between porous and healthy cases. This ranking takes into account the intensity of the stress concentration $K_{t}$ as well as its spread, with the effective volume term $V_{\text {eff }}$.

3. The ranking in pore criticality in this model allows for the consideration of the spread of a heterogeneous stress field, and has a finer sensibility to the position of defects than a Murakami-type approach.

4. A numerical fatigue test campaign methodology is set-up. Numerical samples are extracted from a porous volume characterized by parameters $k$ and $d$. For each sample the SNP curves are interpolated from an FEA pore database. The resulting scattered SNP curves are coherent with experimental results.

5. The proposed methodology allows a critical pore population to be generated from a given initial population for a given geometry. It is found that the values of $k$ and $d$ identified using the fractography data and the model are very close to the tomography results, giving great confidence in the ability of the model to rank pores in fatigue criticality.

The choice was made in this study to consider defects as perfectly spherical, when in reality, multiple critical pores were ellipsoids. The shape and orientation of ellipsoidal pores would constitute new parameters for a finer description of the scatter of fatigue results. The assumption of a random spatial distribution of pores could also be discussed in the case of WAAM structures produced as near-net shape.

The purpose of this methodology is to provide designers with a tool for the dimensioning in fatigue of structural WAAM parts. The exclusion of pores from the intrinsic material properties implies that, if process parameters are to be changed, only tomography analyses are required to characterize the new WAAM material containing a new pore population, thereby avoiding the need for further fatigue tests. As it was demonstrated here with fatigue samples, a draw of numerical structures, or critically stressed zones, could be generated to forecast fatigue performances of industrial parts. From the results, design rules or a defect size reject criterion could be determined.

\section{Acknowledgements}

The authors would like to thank Direction Générale de l'Armement (DGA) for the funding of this study. This work was performed as part of the Gustave Zédé joint lab collaborative research between Naval Group and ENSTA Bretagne. A part of this sutdy belongs to the "Self-Heating" ANR - Safran - Naval Group research chair (Grant \# ANR-20-CHIN-0002) involving Safran Companies, Naval Group, ENSTA Bretagne (IRDL) and Institut Pprime.

\section{References}

[1] M. Orme, I. Madera, M. Gschweitl, and M. Ferrari, "Topology Optimization for Additive Manufacturing as an Enabler for Light Weight Flight Hardware,” Designs, vol. 2, no. 4, p. 51, 2018.

[2] T. Abe and H. Sasahara, "Layer geometry control for the fabrication of lattice structures by wire and arc additive manufacturing," Additive Manufacturing, vol. 28, no. January, pp. 639-648, 2019.

[3] Ivántabernero, A. Paskual, P. Álvarez, and A. Suárez, "Study on Arc Welding Processes for High Deposition Rate Additive Manufacturing," Procedia CIRP, vol. 68, no. April, pp. 358-362, 2018.

[4] S. W. Williams, F. Martina, A. C. Addison, J. Ding, G. Pardal, and P. Colegrove, "Wire + Arc additive manufacturing," Materials Science and Technology (United Kingdom), vol. 32, no. 7, pp. 641-647, 2016.

[5] A. Horgar, H. Fostervoll, B. Nyhus, X. Ren, M. Eriksson, and O. M. Akselsen, "Additive manufacturing using WAAM with AA5183 wire," Journal of Materials Processing Technology, vol. 259, no. November 2017, pp. 68-74, 2018.

[6] D. Ding, Z. Pan, S. van Duin, H. Li, and C. Shen, "Fabricating superior NiAl bronze components through wire arc additive manufacturing," Materials, vol. 9, no. 8, 2016.

[7] J. Xiong, Y. J. Li, Z. Q. Yin, and H. Chen, "Determination of surface roughness in wire and arc additive manufacturing based on laser vision sensing," Chinese Journal of Mechanical Engineering (English Edition), vol. 31, no. 4, pp. 1-7, 2018.

[8] J. Pegues, M. Roach, R. Scott Williamson, and N. Shamsaei, "Surface roughness effects on the fatigue strength of additively manufactured Ti-6Al-4V," International Journal of Fatigue, vol. 116, no. April, pp. 543-552, 2018.

[9] B. Vayssette, N. Saintier, C. Brugger, M. Elmay, and E. Pessard, "Surface roughness of Ti-6Al-4V parts obtained by SLM and EBM: Effect on the High Cycle Fatigue life,” Procedia Engineering, vol. 213, pp. 89-97, 2018. 
[10] J. R. Hönnige, P. A. Colegrove, B. Ahmad, M. E. Fitzpatrick, S. Ganguly, T. L. Lee, and S. W. Williams, "Residual stress and texture control in Ti-6Al-4V wire + arc additively manufactured intersections by stress relief and rolling," Materials and Design, vol. 150, pp. 193-205, 2018.

[11] B. A. Szost, S. Terzi, F. Martina, D. Boisselier, A. Prytuliak, T. Pirling, M. Hofmann, and D. J. Jarvis, “A comparative study of additive manufacturing techniques: Residual stress and microstructural analysis of CLAD and WAAM printed Ti-6Al-4V components," Materials and Design, vol. 89, pp. 559-567, 2016.

[12] E. M. Ryan, T. J. Sabin, J. F. Watts, and M. J. Whiting, "The influence of build parameters and wire batch on porosity of wire and arc additive manufactured aluminium alloy 2319," Journal of Materials Processing Technology, vol. 262, no. April, pp. 577-584, 2018.

[13] F. Wang, S. Williams, P. Colegrove, and A. A. Antonysamy, "Microstructure and mechanical properties of wire and arc additive manufactured Ti-6Al-4V," Metallurgical and Materials Transactions A: Physical Metallurgy and Materials Science, vol. 44, no. 2, pp. 968-977, 2013.

[14] S. Siddique, M. Imran, E. Wycisk, C. Emmelmann, and F. Walther, "Influence of process-induced microstructure and imperfections on mechanical properties of AlSi12 processed by selective laser melting," Journal of Materials Processing Technology, vol. 221, pp. 205-213, 2015.

[15] S. Siddique, M. Imran, and F. Walther, "Very high cycle fatigue and fatigue crack propagation behavior of selective laser melted AlSi12 alloy," International Journal of Fatigue, vol. 94, pp. 246-254, 2017.

[16] Y. N. Hu, S. C. Wu, P. J. Withers, J. Zhang, H. Y. Bao, Y. N. Fu, and G. Z. Kang, "The effect of manufacturing defects on the fatigue life of selective laser melted Ti-6Al-4V structures," Materials and Design, vol. 192, no. July, 2020.

[17] C. Douellou, X. Balandraud, E. Duc, B. Verquin, F. Lefebvre, and F. Sar, "Rapid characterization of the fatigue limit of additivemanufactured maraging steels using infrared measurements," Additive Manufacturing, no. May, p. 101310, 2020.

[18] V. D. Le, E. Pessard, F. Morel, and F. Edy, "Interpretation of the fatigue anisotropy of additively manufactured TA6V alloys via a fracture mechanics approach," Engineering Fracture Mechanics, vol. 214, pp. 410-426, 2019.

[19] Y. Murakami and M. Endo, "Effects of defects, inclusions and inhomogeneities on fatigue strength," International Journal of Fatigue, vol. 16, no. 3, pp. 163-182, 1994.

[20] H. Danninger and B. Weiss, “The influence of defects on high cycle fatigue of metallic materials," Journal of Materials Processing Technology, vol. 143-144, no. 1, pp. 179-184, 2003.

[21] S. Leuders, M. Vollmer, F. Brenne, T. Tröster, and T. Niendorf, "Fatigue Strength Prediction for Titanium Alloy TiAl6V4 Manufactured by Selective Laser Melting," Metallurgical and Materials Transactions A: Physical Metallurgy and Materials Science, vol. 46, no. 9, pp. 3816-3823, 2015.

[22] S. Tammas-Williams, J. Donoghue, P. J. Withers, I. Todd, and P. Prangnell, "Predicting the Influence of Porosity on the Fatigue Performance of Titanium Components Manufactured by Selective Electron Beam Melting," Proceedings of the 13th World Conference on Titanium, pp. 1447-1452, 2016.

[23] R. Biswal, X. Zhang, M. Shamir, A. Al Mamun, M. Awd, F. Walther, and A. Khadar Syed, "Interrupted fatigue testing with periodic tomography to monitor porosity defects in wire + arc additive manufactured Ti-6Al-4V," Additive Manufacturing, vol. 28, no. April, pp. 517-527, 2019.

[24] K. S. Derekar, A. Addison, S. S. Joshi, X. Zhang, J. Lawrence, L. Xu, G. Melton, and D. Griffiths, "Effect of pulsed metal inert gas (pulsed-MIG) and cold metal transfer (CMT) techniques on hydrogen dissolution in wire arc additive manufacturing (WAAM) of aluminium," International Journal of Advanced Manufacturing Technology, vol. 107, no. 1-2, pp. 311-331, 2020.

[25] J. Wang, Y. Cui, C. Liu, Z. Li, Q. Wu, and D. Fang, "Understanding internal defects in Mo fabricated by wire arc additive manufacturing through 3D computed tomography," Journal of Alloys and Compounds, vol. 840, p. $155753,2020$.

[26] R. Biswal, A. K. Syed, and X. Zhang, "Assessment of the effect of isolated porosity defects on the fatigue performance of additive manufactured titanium alloy," Additive Manufacturing, vol. 23, no. July, pp. 433-442, 2018.

[27] E. Wycisk, A. Solbach, S. Siddique, D. Herzog, F. Walther, and C. Emmelmann, "Effects of defects in laser additive manufactured Ti-6Al-4V on fatigue properties,” Physics Procedia, vol. 56, no. C, pp. 371-378, 2014.

[28] B. Cong, J. Ding, and S. Williams, "Effect of arc mode in cold metal transfer process on porosity of additively manufactured Al-6.3\%Cu alloy," International Journal of Advanced Manufacturing Technology, vol. 76, no. 9-12, pp. 1593-1606, 2015.

[29] B. Cong, Z. Qi, B. Qi, H. Sun, G. Zhao, and J. Ding, “A comparative study of additively manufactured thin wall and block structure with Al-6.3\% Cu alloy using cold metal transfer process," Applied Sciences (Switzerland), vol. 7, no. 3, 2017. 
[30] O. Panchenko, D. Kurushkin, I. Mushnikov, A. Khismatullin, and A. Popovich, "A high-performance WAAM process for Al-Mg-Mn using controlled short-circuiting metal transfer at increased wire feed rate and increased travel speed," Materials and Design, vol. 195, p. 109040, 2020.

[31] R. Biswal, X. Zhang, A. K. Syed, M. Awd, J. Ding, F. Walther, and S. Williams, "Criticality of porosity defects on the fatigue performance of wire + arc additive manufactured titanium alloy," International Journal of Fatigue, vol. 122, no. September 2018, pp. 208-217, 2019. [Online]. Available: https://doi.org/10.1016/j.ijfatigue.2019.01.017

[32] ASTM E466-15, "Standard Practice for Conducting Force Controlled Constant Amplitude Axial Fatigue Tests of Metallic Materials," ASTM International, West Conshohocken, PA, 2015. [Online]. Available: www.astm.org

[33] W. J. Dixon and A. M. Mood, “A Method for Obtaining and Analyzing Sensitivity Data," Journal of the American Statistical Association, vol. 43, no. 241, pp. 109-126, 1948.

[34] H. Özdeş, M. Tiryakioğlu, and P. D. Eason, "On estimating axial high cycle fatigue behavior by rotating beam fatigue testing: Application to A356 aluminum alloy castings," Materials Science and Engineering A, vol. 697, no. March, pp. 95-100, 2017.

[35] S. Romano, A. Brückner-Foit, A. Brandão, J. Gumpinger, T. Ghidini, and S. Beretta, "Fatigue properties of AlSi10Mg obtained by additive manufacturing: Defect-based modelling and prediction of fatigue strength," Engineering Fracture Mechanics, vol. 187, pp. 165-189, 2018. [Online]. Available: https://doi.org/10.1016/j.engfracmech.2017.11.002

[36] S. Siddique, M. Awd, J. Tenkamp, and F. Walther, "Development of a stochastic approach for fatigue life prediction of AlSi12 alloy processed by selective laser melting," Engineering Failure Analysis, vol. 79, pp. 34-50, 2017. [Online]. Available: http://dx.doi.org/10.1016/j.engfailanal.2017.03.015

[37] C. E. Stromeyer, “The determination of fatigue limits under alternating stress conditions," Proceedings of the Royal Society of London, vol. 90, no. 620, pp. 411-425, 1914.

[38] G. Fargione, A. Geraci, G. La Rosa, and A. Risitano, "Rapid determination of the fatigue curve by the thermographic method," International Journal of Fatigue, vol. 24, no. 1, pp. 11-19, 2002.

[39] J.-C. Krapez and D. Pacou, "Thermography detection of early thermal effects during fatigue tests of steel and aluminum samples," AIP Conference Proceedings, vol. 615, pp. 1545-1552, 2002.

[40] C. Doudard, S. Calloch, P. Cugy, A. Galtier, F. Hild, C. Doudard, S. Calloch, P. Cugy, A. Galtier, and F. H. A. Probabilistic, “A probabilistic two-scale model for high-cycle fatigue life predictions," Fatigue \& Fracture of Engineering Materials \& Structures, vol. 28, pp. 279-288, 2005.

[41] R. Munier, C. Doudard, S. Calloch, and B. Weber, "Determination of high cycle fatigue properties of a wide range of steel sheet grades from self-heating measurements,” International Journal of Fatigue, vol. 63, pp. 46-61, 2014.

[42] J. Ge, J. Lin, H. Fu, Y. Lei, and R. Xiao, “A spatial periodicity of microstructural evolution and anti-indentation properties of wire-arc additive manufacturing 2Cr13 thin-wall part," Materials and Design, vol. 160, no. 100, pp. 218-228, 2018.

[43] A. B. Kale, J. Singh, B.-K. Kim, D.-I. Kim, and S.-H. Choi, "Effect of initial microstructure on the deformation heterogeneities of 316L stainless steels fabricated by selective laser melting processing," Journal of Materials Research and Technology, vol. 9, no. 4, pp. 8867-8883, 2020.

[44] S. Kiakidis, "Mechanical and corrosion behaviour of 3D printed aluminium bronzes produced by wire+arc additive manufacturing: In collaboration with RAMLAB," Ph.D. dissertation, Delft University, 2017.

[45] I. Richardson, "Guide to Nickel Aluminium Bronze for Engineers," p. 100, 2016.

[46] Y. Lv, L. Wang, X. Xu, Y. Han, and W. Lu, "Investigation of the microstructure and corrosion properties of friction stir processed cast NiAl bronze," Materials Transactions, vol. 56, no. 9, pp. 1523-1529, 2015.

[47] F. Hasan, A. Jahanafrooz, G. W. Lorimer, and N. Ridley, "MORPHOLOGY, CRYSTALLOGRAPHY, AND CHEMISTRY OF PHASES IN As-CAST NICKEL-ALUMINUM BRONZE.” Metallurgical transactions. A, Physical metallurgy and materials science, vol. 13 A, no. 8, pp. 1337-1345, 1982.

[48] X. Xu, Y. Lv, M. Hu, D. Xiong, L. Zhang, L. Wang, and W. Lu, "Influence of second phases on fatigue crack growth behavior of nickel aluminum bronze," International Journal of Fatigue, vol. 82, pp. 579-587, 2016.

[49] A. Ezanno, C. Doudard, S. Calloch, and J. L. Heuzé, "A new approach to characterizing and modeling the high cycle fatigue properties of cast materials based on self-heating measurements under cyclic loadings," International Journal of Fatigue, vol. 47, pp. 232-243, 2013.

[50] C. Caër, L. Bercelli, C. Doudard, S. Calloch, M. Dhondt, and J. Beaudet, "Caractérisation microstructurale et mécanique d'un acier Duplex issu de la fabrication additive," in CFM 2019, 2019. [Online]. Available: https://cfm2019.sciencesconf.org/254844 
[51] A. Queguineur, J. Marolleau, A. Lavergne, and G. Rückert, "Evaluation of tandem controlled short-circuit GMAW for improved deposition in additive manufacture of large Nickel Aluminium Bronze naval components," Welding in the World, vol. 64, no. 8, pp. 1389-1395, 2020.

[52] Z. Qiu, B. Wu, H. Zhu, Z. Wang, A. Hellier, Y. Ma, H. Li, O. Muransky, and D. Wexler, "Microstructure and mechanical properties of wire arc additively manufactured Hastelloy C276 alloy," Materials and Design, vol. 195, p. 109007, 2020.

[53] N. A03-400, "Produits sidérurgiques - Essais de fatigue - Principes généraux," 1983.

[54] N. A03-401, "Produits sidérurgiques - Essai de fatigue par charge axiale," 1983.

[55] S. Tammas-Williams, P. J. Withers, I. Todd, and P. B. Prangnell, "The Influence of Porosity on Fatigue Crack Initiation in Additively Manufactured Titanium Components," Scientific Reports, vol. 7, no. 1, 2017.

[56] V. D. Le, E. Pessard, F. Morel, and F. Edy, "Influence of porosity on the fatigue behaviour of additively fabricated TA6V alloys," MATEC Web of Conferences, vol. 165, no. January 2019, 2018.

[57] S. Romano, A. Brandão, J. Gumpinger, M. Gschweitl, and S. Beretta, "Qualification of AM parts: Extreme value statistics applied to tomographic measurements," Materials and Design, vol. 131, no. April, pp. 32-48, 2017.

[58] J. Louge, C. Doudard, S. Calloch, and B. Weber, "Study of load history effects on the high cycle fatigue properties of high-strength low-alloy steel from self-heating measurements," MATEC Web of Conferences, vol. 165, pp. 1-7, 2018.

[59] J. O. Smith, “The Effect of Range of Stress on the Fatigue Strength of Metals," University of Illinois Bulletin, vol. XXXIX, no. 334, pp. 7-52, 1942.

[60] M. Poncelet, C. Doudard, S. Calloch, B. Weber, and F. Hild, "Probabilistic multiscale models and measurements of self-heating under multiaxial high cycle fatigue,” Journal of the Mechanics and Physics of Solids, vol. 58, no. 4, pp. 578-593, 2010.

[61] R. Munier, C. Doudard, S. Calloch, and B. Weber, "Identification of the micro-plasticity mechanisms at the origin of self-heating under cyclic loading with low stress amplitude," International Journal of Fatigue, vol. 103, pp. 122-135, 2017.

[62] W. Weibull, “A Statistical Distribution Function of Wide Applicability,” Journal of Applied Mechanics, vol. 13, pp. 293-297, 1951.

[63] E. Charkaluk, A. Bignonnet, A. Constantinescu, and K. Dang Van, "Fatigue design of structures under thermomechanical loadings," Fatigue and Fracture of Engineering Materials and Structures, vol. 25, no. 12, pp. 1199-1206, 2002. 